\title{
Entanglement dynamics in short and long-range harmonic oscillators
}

\author{
M. Ghasemi Nezhadhaghighi ${ }^{1}$ and M. A. Rajabpour ${ }^{2,3, *}$ \\ ${ }^{1}$ Department of Physics, College of Sciences, Shiraz University, Shiraz 71454, Iran \\ ${ }^{2}$ Instituto de Física de São Carlos, Universidade de São Paulo, \\ Caixa Postal 369, 13560-970 São Carlos, SP, Brazil \\ ${ }^{3}$ Instituto de Física, Universidade Federal Fluminense, \\ Av. Gal. Milton Tavares de Souza s/n, Gragoatá, 24210-346, Niterói, RJ, Brazil.
}

\begin{abstract}
We study the time evolution of the entanglement entropy in the short and long-range coupled harmonic oscillators that have well-defined continuum limit field theories. We first introduce a method to calculate the entanglement evolution in generic coupled harmonic oscillators after quantum quench. Then we study the entanglement evolution after quantum quench in harmonic systems that the couplings decay effectively as $1 / r^{d+\alpha}$ with the distance $r$. After quenching the mass from non-zero value to zero we calculate numerically the time evolution of von Neumann and Rényi entropies. We show that for $1<\alpha<2$ we have a linear growth of entanglement and then saturation independent of the initial state. For $0<\alpha<1$ depending on the initial state we can have logarithmic growth or just fluctuation of entanglement. We also calculate the mutual information dynamics of two separated individual harmonic oscillators. Our findings suggest that in our system there is no particular connection between having a linear growth of entanglement after quantum quench and having a maximum group velocity or generalized Lieb-Robinson bound.
\end{abstract}

Contents

\section{Introduction}

II. Basic Definitions

III. Quantum quench

IV. Harmonic systems with local couplings

V. Harmonic systems with long-range couplings

A. Weakly coupled long-range harmonic oscillators $1<\alpha<2$

B. Strongly coupled long-range harmonic oscillators $0<\alpha<1$

VI. Initial state effects

A. Gapless power-law initial state

B. Exponential decaying initial state

C. Unentangled initial state

VII. Mutual information dynamics

VIII. Conclusion and remarks

Acknowledgments

A. Gapfull power-law initial state

B. Details on numerical calculations

References

*Electronic address: rajabpour@ursa.ifsc.usp.br

\section{INTRODUCTION}

1 There has been considerable interest in the entanglement properties of quantum many body systems from dif-

3 ferent point of views i.e. quantum field theory [1] , quantum phase transition [2] and quantum information theory

3 3]. Among the various measures of quantifying quantum entanglement entropy, the von Neumann and Rényi en-

5 tropies, have been studied in many different locally coupled systems [4 9]. At the quantum critical point, conformal field theory (CFT) predicts that the entanglement 7 entropy in one spatial dimension scales logarithmically with respect to the size of the subsystem $l$. If the total 8 system is infinitely long, $S_{l}=\frac{c}{3} \log l$ where $c$ is the central charge of the CFT [1]. Away from the critical point,

10 the entropy for blocks larger than the spatial correlation length $\xi \sim m^{-1}$ saturates to a value $S_{l}=-\frac{c}{3} \log m$ where $11 m$ is the energy gap of the system [6]. For general free 12 quantum field theories in the case of higher dimensions, it 12 has been shown that the von Neumann entropy satisfies 14 the area law. Based on the area law the entanglement entropy is proportional to the interface area between the subsystem and its complement [10, 11].

Although many studies have dealt with the short-range 17 systems and numerous results have been discovered in the last few years, a less investigated situation is how (one 17 measure of) quantum entanglement scales with the subsystem size when the couplings in the model are long-

17 range. The entanglement entropy of one dimensional models with long-range couplings have been studied so

17 far in the following examples: the Lipkin-Meshkov-Glick (LMG) model which in that all spins interact among 20 themselves [14, 15], the long-range Ising type model [16], the anti-ferromagnetic long-range Ising chain [17] and free fermions with long-range unshielded Coulomb interaction [18]. One of the most important features of the long-range systems is the presence of a power-law like 
dispersion relation, i.e. $\omega^{2}(k) \sim|k|^{\alpha}$ with $0<\alpha<2$ [19]. The same is true for those harmonic oscillators that have $1 /|r|^{d+\alpha}$ like couplings. It is widely believed that it should be true also for spin systems with long-range interactions 20, 21].

In the sense of universality, quantum phase transition in spin systems with long-range interaction is generally described, via thermal phase transitions in an equivalent classical $(d+1)$-dimensional spin model with long-range interaction in $d$-spatial dimensions and short-range interaction in the $(d+1)$-th dimension. In the specific case where a long-range couplings falling off as $1 /|r|^{d+\alpha}$, if $\alpha \geq 2$ the corresponding classical $(d+1)$-dimensional spin model describes the short-range quantum models. It is important to note that based on the results were given in Ref. 20], the upper critical dimension for such systems is given by $d_{u}=3 \alpha / 2$. For example, the quantum transitions of one-dimensional quantum transverse Ising model with long-range interaction with values of $\alpha \leq 2 / 3$ are described by long-range mean-field theory [20]. Therefore, the scaling limit of such systems with long-range couplings "particularly in some regions" can be described by free fractional field theories. Recently, in Refs. 22] and [23], we studied the entanglement entropy of a block of long-range coupled harmonic oscillators. A crucial point is that we showed the entanglement of the gapless $1 d$ system is logarithmically dependent on the subsystem size and we measured the prefactor of the logarithm in different situations. In higher dimensions we show the von Neumann entropy of a partition scales with the surface area.

In principle our formalism is based on the first quantization of the free fractional field theories. This method is first discussed by Bombelli, et.al 24] which they compute the entanglement entropy of free field theory by using the discrete version of the field theory which is simply coupled harmonic oscillators making it easy to numerically evaluate. It is worth mentioning that [26] and [27], studied a certain class of non-local field theories whose ground state entanglement entropy follows a volume law.

The past few years have witnessed a renewed interest in the the experimental methods that have been proposed in the study of isolated quantum systems i.e. optically trapped ultra-cold atomic gases, which they rely on probing the non-equilibrium properties of the system [28]. From a theoretical point of view, the non-equilibrium dynamics after a sudden change (quench) of a parameter in the hamiltonian of the quantum system is one of the most remarkable aspects of these studies. For example, consider a quantum system with hamiltonian $H(m)$ depending on a parameter $m$. The system is prepared in a pure state (eigenstate) of a given hamiltonian $H\left(m_{0}\right)$. Then, at time $t=0$ the parameter is suddenly quenched from $m_{0}$ to $m$. After the quench, there is an extensive excess in energy which appear as quasiparticles that propagate in time 29].

The qualitative, and quantitative, features found for the non-equilibrium dynamics of quantum systems may be understood by the time evolution of the entanglement entropy. In this investigation one asks how the entanglement evolves in time after the quantum quench. The most remarkable results that emerged from theoretical investigations is that; if the hamiltonian $H$ governing the time evolution is at a critical point, the entanglement entropy $S_{A}(t)$ grows linearly with time $t$, up to $t \sim l / 2$ where $l$ is the subsystem size. Thereafter it immediately saturates [29]. The same linear behavior for the growth of entanglement entropy, has been also shown for a free quantum field theory in $d+1$-dimensions [30, 31].

As it is well known, a lot of numerical and theoretical works have been done seeking to understand the mechanism of the time evolution of the quantum entanglement in quantum systems [32 [39, 46] $]$. Recent theoretical works have investigated the existence of quasiparticles or longwavelength propagating modes such as acoustic sound following a quantum quench [29, 40]. In real physical systems, with local interaction, one might expect that, there is an upper limit on the velocity of propagating information (such as energy quasiparticles). This limit is a consequence of the Lieb-Robinson (LR) bounds [41]. Based on the LR theorem, the propagation of perturbations, cannot spread faster than Lieb-Robinson velocity. The existence of such bound corresponds with a horizon like region defined by this velocity, which inside of it the correlations are non-zero and outside they are exponentially suppressed [42]. This effect in non-relativistic quantum many-body systems is usually called quasi-locality which plays the same role as the velocity of light in the Lorentz invariant field theories. Lieb-Robinson bounds can be used to show that there exists an upper bound for the amount of entanglement that can be produced in a finite time after quench which increases linearly in time [40, 43].

An important question to ask about entanglement dynamics is what kind of scaling with time can one expect for the entanglement entropy of quantum systems with long-range couplings? The first numerical studies of entanglement dynamics generated by long-range interactions appeared in Refs. 44]. It is worth mentioning that there are some considerable experimental results available about the propagation of the correlations through the quantum many-body system with long-range interactions [45, 46]. Specifically, in Ref. 44] they explored entanglement entropy and mutual information dynamics after a quantum quench in the transverse field Ising model with long-range interactions, which can be described by the hamiltonian $H=\sum_{i<j} J_{i, j} \hat{\sigma}_{i}^{x} \hat{\sigma}_{j}^{x}+B \sum_{i} \hat{\sigma}_{i}^{z}$ and the couplings decay with distance like $J_{i, j} \propto|i-j|^{-\sigma}$ $(\sigma>0)$. Interestingly, for those long-range interactions with $\sigma>1$, they found that the behavior of the time evolution of entanglement entropy is qualitatively similar to nearest-neighbor interactions. Remarkably, they also found that, for interaction exponent $\sigma<1$, the growth of entanglement is only logarithmic.

It is worth mentioning that there are also some interesting results available to extend the Lieb and Robinson 
theory for quantum systems with power-law $\left(\sim 1 / r^{\sigma}\right)$ decaying couplings with $\sigma>d$ [47, 48]. Unlike the systems with only short-range interactions, the locality picture for quantum systems with long range couplings when the interaction exhibits a power-law decay with an exponent $\sigma<d$, is no-longer true. There is no concrete result for the special behavior of the growth and propagation of entanglement entropy in quantum systems with long-range couplings. Currently, the question of extension of Lieb-Robinson theorem to different quantum systems with long-range interactions is a subject of active theoretical and numerical debate [49 52].

The purpose of this manuscript is to describe an efficient computational method to study the time evolution of the entanglement entropy for coupled long-range harmonic oscillators in any dimension. The structure of the rest of the paper is as follows. In section II we will first introduce our system. In section [II] we introduce a method to numerically evaluate the time evolution of von-Neumann and Rényi entropies in harmonic oscillators and we outline the setup for the global quench which is then applied to different situations in the following sections. In section IV we numerically study the entanglement entropy dynamics for harmonic oscillators with short-range couplings. In section $\nabla$ we evaluated the same analysis when the harmonic system is long-range. In section $\nabla \mathbf{V I}$ we analyzed the initial state effect on the entanglement dynamics. In section VII] we studied different aspects of the mutual information propagation in long-range harmonic oscillators after global quench. Finally, in section VIII we provide a conclusion. To be self-explanatory, in the appendix we will give more details about our numerical methods.

\section{BASIC DEFINITIONS}

Consider a one-dimensional system of $N$ bosonic oscillators. They are coupled by a quadratic hamiltonian of the form

$$
\mathcal{H}=\frac{1}{2} \sum_{n=1}^{N} \pi_{n}^{2}+\frac{1}{2} \sum_{n, n^{\prime}=1}^{N} \phi_{n} K_{n n^{\prime}} \phi_{n^{\prime}},
$$

where the kernel matrix $K$ is real and positive semidefinite due to the hermiticity and $\left\{\phi_{i}\right\}$ is a scalar field. The normalized ground state wave function for such system is given as gaussian state

$$
\Psi_{0}(\{\phi\}) \propto(\operatorname{det} \Gamma)^{\frac{1}{4}} \exp \left\{-\frac{1}{2} \sum_{n, n^{\prime}=1}^{N} \phi_{n} \Gamma_{n n^{\prime}} \phi_{n^{\prime}}\right\},
$$

where $\{\phi\}$ denotes the collection of all $\phi$ 's, one for each oscillator and $\Gamma=K^{1 / 2}$. Having the solution of the ground state wave function one can calculate the reduced density matrix of a block of $l$ oscillators. Consider a system which is divided into two subsystems $A$ and $B$ with sizes $l$ and $N-l$ respectively. Suppose the whole system is in a pure quantum state, with density matrix $\rho$. One can obtain $A$ 's reduced density matrix by tracing out the remaining degrees of freedom $\rho_{A}=\operatorname{tr}_{B} \rho$.

There are several measures of entanglement between parties of a closed system, examples being the von Neumann and Rényi entropies. The entanglement entropy associated to the local density matrix $\rho_{A}$ is just the von Neumann entropy

$$
S_{A}=-\operatorname{tr}\left(\rho_{A} \log \left(\rho_{A}\right)\right) .
$$

The von Neumann entropy is the most well-known member of a more general family of entanglement entropies, the so-called Reńyi entropies, defined as

$$
S_{n}(A)=\frac{1}{1-n} \log \left(\operatorname{tr} \rho_{A}^{n}\right), \quad n \geq 0, \quad n \neq 1 .
$$

The Rényi entropy reduces to the von Neumann entropy when $n \rightarrow 1\left(S_{A}=\lim _{n \rightarrow 1} S_{n}(A)\right)$.

In the next section we will present a detailed study of the time evolution of von Neumann and Rényi entropies that results after a quantum quench in a quantum system.

\section{QUANTUM QUENCH}

The purpose of this section is to describe an efficient method to study the time-dependent behavior of the entanglement entropy after a global changing of a parameter in the system in any spatial dimension, also known as quantum quench.

Here we consider first that the system is prepared in the ground state of a given gapped hamiltonian $H(m)$, where $m$ is tuneable parameter. At time $t=0$ the parameter $m_{0}$ is changed suddenly to a different value $m$. For $t>0$ the system is allowed to evolve with a different hamiltonian $H(m)$. In this study we will consider $m=0$. To study the entanglement dynamics for the systems with global quench the only thing that we need is to start with an arbitrary gaussian state and then see its evolution with respect to the new hamiltonian. Since the hamiltonian of the system is quadratic, one can expect that, the time-evolved state remains gaussian in the form

$$
\Psi(\{\phi\}, t) \propto(\operatorname{det} \tilde{A})^{\frac{1}{4}} \exp \left\{-\frac{1}{2} \sum_{n, n^{\prime}=1}^{N} \phi_{n} A_{n n^{\prime}}(t) \phi_{n^{\prime}}\right\},
$$

where a tilde denotes the real part. It is easy to show that $A(t)$ obeys the Riccati equation

$$
i \frac{\partial A}{\partial t}=A^{2}-K, \quad A(0)=\Gamma .
$$

Its solution is given by

$$
A(t)=K^{1 / 2} \frac{\cos \left(t K^{1 / 2}\right) \Gamma+i K^{1 / 2} \sin \left(t K^{1 / 2}\right)}{\cos \left(t K^{1 / 2}\right) K^{1 / 2}+i \sin \left(t K^{1 / 2}\right) \Gamma},
$$


where $K$ is the hamiltonian after quench and the matrix $\Gamma=K_{0}^{1 / 2}$ is prepared at $t=0$.

Having the above equations one can simply use the following techniques to calculate the dynamics of entanglement entropy. Suppose we cut a harmonic chain at the boundaries between two subsystems $A$ and $B$. Now consider

$$
A^{-1}(t)=\left(\begin{array}{cc}
X_{A} & X_{A B} \\
X_{A B}^{T} & X_{B}
\end{array}\right), \quad A(t)=\left(\begin{array}{cc}
P_{A} & P_{A B} \\
P_{A B}^{T} & P_{B}
\end{array}\right)
$$

where $X_{A}\left(P_{A}\right)$ is $l \times l$ and $X_{B}\left(P_{B}\right)$ is $(N-l) \times(N-l)$ matrix. Then one can write the reduced density matrix as 36.

$$
\begin{aligned}
\rho_{B}\left(\left\{\phi^{1}\right\}_{B} ;\left\{\phi^{2}\right\}_{B}\right) \propto & \propto \exp \left\{-\frac{1}{2}\left(\left\{\phi^{1}\right\}_{B}\left\{\phi^{2}\right\}_{B}\right) \times\right. \\
& \left.\left(\begin{array}{cc}
\mathcal{C} & 2 \mathcal{D} \\
2 \mathcal{D}^{*} & \mathcal{C}^{*}
\end{array}\right)\left(\begin{array}{l}
\left\{\phi^{1}\right\}_{B} \\
\left\{\phi^{2}\right\}_{B}
\end{array}\right)\right\},
\end{aligned}
$$

where we have

$$
\begin{aligned}
\mathcal{C} & =P_{B}-\frac{P_{A B}^{T}\left(\tilde{P}_{A}\right)^{-1} P_{A B}}{2} \\
\mathcal{D} & =-\frac{P_{A B}^{T}\left(\tilde{P}_{A}\right)^{-1} P_{A B}^{*}}{4}
\end{aligned}
$$

with $\tilde{M}=\left(M+M^{*}\right) / 2$.

We will now follow the method was first introduced in [24] and then elaborated in [53], to get the von Neumann and Rényi entropies. Using Eq. (9) and the method followed in [53] we investigated the trace of $n$-th power of the reduced density matrix as a functional integral:

$$
\begin{gathered}
\operatorname{tr} \rho_{B}^{n}=\int\left[\prod_{m \in(B)} d \phi_{m}\right] \exp \left\{-\left(\left\{\phi^{1}\right\}_{B} \cdots\left\{\phi^{n}\right\}_{B}\right) \times\right. \\
\left.\mathcal{M}_{n}\left(\begin{array}{c}
\left\{\phi^{1}\right\}_{B} \\
\vdots \\
\left\{\phi^{n}\right\}_{B}
\end{array}\right)\right\}
\end{gathered}
$$

where $\mathcal{M}_{n}$ is the matrix

$$
\mathcal{M}_{n}=\left(\begin{array}{cccccc}
\tilde{\mathcal{C}} & \mathcal{D} & 0 & \ldots & 0 & \mathcal{D}^{*} \\
\mathcal{D}^{*} & \tilde{\mathcal{C}} & \mathcal{D} & \ldots & 0 & 0 \\
0 & \mathcal{D}^{*} & \tilde{\mathcal{C}} & \ldots & 0 & 0 \\
\vdots & \vdots & \vdots & & \vdots & \vdots \\
0 & 0 & 0 & \ldots & \tilde{\mathcal{C}} & \mathcal{D} \\
\mathcal{D} & 0 & 0 & \ldots & \mathcal{D}^{*} & \tilde{\mathcal{C}}
\end{array}\right)
$$

Two matrices $\mathcal{C}$ and $\mathcal{D}$ are defined in the Eq. (10). It is worth mentioning that the Eq. (11) is a gaussian functional integral, therefore it is possible to find the result $\operatorname{tr} \rho^{n}=1 / \sqrt{\operatorname{det} \mathcal{M}_{n}}$ but there is no straight-forward way to compute this determinant as a function of $n$, however, one can find the result by rescaling the density matrix. Consider the new matrix

$$
\mathcal{M}_{n}^{\prime}=\left(\begin{array}{cccccc}
2 & -\mathcal{E} & 0 & \ldots & 0 & -\mathcal{E}^{*} \\
-\mathcal{E}^{*} & 2 & -\mathcal{E} & \ldots & 0 & 0 \\
0 & -\mathcal{E}^{*} & 2 & \ldots & 0 & 0 \\
\vdots & \vdots & \vdots & & \vdots & \vdots \\
0 & 0 & 0 & \ldots & 2 & -\mathcal{E} \\
-\mathcal{E} & 0 & 0 & \ldots & -\mathcal{E}^{*} & 2
\end{array}\right)
$$

where $\mathcal{E}=-2(\tilde{\mathcal{C}})^{-1} \mathcal{D}$. By taking into account $\operatorname{det} \mathcal{M}_{n}^{\prime}=$ $2^{n} \operatorname{det} \mathcal{M}_{n} /(\operatorname{det} \tilde{\mathcal{C}})^{n}$ the trace of the power $n$ of the reduced density matrix is then

$$
\operatorname{tr} \rho_{B}^{n} \propto \frac{1}{\sqrt{\operatorname{det} \mathcal{M}_{n}^{\prime}}}
$$

Let us note that it is possible to consider the matrix $\mathcal{E}$ as a parameter to explicitly diagonalize the matrix $\mathcal{M}_{n}^{\prime}$ and give the result as

$$
\operatorname{det} \mathcal{M}_{n}^{\prime}=\prod_{j=1}^{n}\left(2-2 \mathcal{E} \omega_{j}-2 \mathcal{E}^{*} \omega_{j}^{n-1}\right),
$$

with $\omega_{j}=e^{2 \pi i j / n}$. One can also write the above equation in the form

$$
\operatorname{det} \mathcal{M}_{n}^{\prime}=\prod_{j=1}^{n}\left(2-2|\mathcal{E}| \cos \left(\frac{2 \pi j}{n}+\theta\right)\right),
$$

where $\mathcal{E}=|\mathcal{E}| e^{i \theta}$. Define $|\mathcal{E}|=\frac{2 \xi}{\xi^{2}+1}$ and use the equality

$$
\begin{array}{r}
\prod_{j=0}^{n-1}\left(1+\xi^{2} \quad-2 \xi \cos \left(\theta+\frac{2 \pi j}{n}\right)\right)= \\
1-2 \xi^{n} \cos (n \theta)+\xi^{2 n} .
\end{array}
$$

As has been pointed out above, the operator $\mathcal{E}$ was considered as a number to explicitly diagonalize the matrix $\mathcal{M}_{n}^{\prime}$ and calculate the Eq. (16). But $\mathcal{E}$ is actually an operator, not a number, but we may diagonalize it and apply the above argument for all the eigenvalues. Finally we will have

$$
\operatorname{det} \mathcal{M}_{n}^{\prime}=2^{n} \prod_{\mathcal{E}_{j}} \frac{1-2 \xi^{n}\left(\mathcal{E}_{j}\right) \cos \left(n \theta_{j}\right)+\xi^{2 n}\left(\mathcal{E}_{j}\right)}{\left(1+\xi^{2}\left(\mathcal{E}_{j}\right)\right)^{n}}
$$

with $\mathcal{E}_{j} e^{i \theta_{j}}$ as the eigenvalue of the matrix $\mathcal{E}$. We can now calculate the entanglement entropy by the following formula

$$
S_{A}=\left.\left(-\frac{d}{d n}+1\right) \log \operatorname{tr} \rho_{A}^{n}\right|_{n=1} .
$$

Using Eq. (16), one can write the entropy as a sum over contributions from each eigenvalue of $\mathcal{E}$

$$
\begin{array}{r}
S_{A}=-\sum_{j=1}^{l}\left[\frac{\xi_{j} \log \xi_{j} \cos \theta_{j}-\xi_{j} \theta_{j} \sin \theta_{j}-\xi_{j}^{2} \log \xi_{j}}{1-2 \xi_{j} \cos \theta_{j}+\xi_{j}^{2}}\right. \\
\left.+\frac{1}{2} \log \left(1-2 \xi_{j} \cos \theta_{j}+\xi_{j}^{2}\right)\right],
\end{array}
$$


where $l$ is the size of the subsystem $A$. We should note that Eq. (20) at $t=0$ follows

$$
S_{A}=-\sum_{j=1}^{l}\left[\ln \left(1-\xi_{j}\right)+\frac{\xi_{j}}{1-\xi_{j}} \ln \xi_{j}\right],
$$

in agreement with Ref. [53].

It is also straightforward to write the Rényi entropy $S_{n}$ in terms of $\xi_{j}$ and $\theta_{j}$ as:

$$
\begin{gathered}
S_{n}=\frac{1}{2(n-1)} \sum_{j=1}^{l}\left(\log \left(1-2 \xi_{j}^{2} \cos \left(n \theta_{j}\right)+\xi_{j}^{2 n}\right)\right. \\
\left.-n \log \left(1-2 \xi_{j} \cos \theta_{j}+\xi_{j}^{2}\right)\right) .
\end{gathered}
$$

Note that, the findings presented in Eqs. (20) and (22) are our main result of this section. In the next sections we will numerically evaluate the time evolution of von Neumann and Rényi entropies using Eqs. (20) and (22) and compare the numerical results with the analytical predictions.

\section{HARMONIC SYSTEMS WITH LOCAL COUPLINGS}

In this section we discuss numerical evaluation of the dynamics of entanglement entropy $S_{A}(t)$ and the Rényi entropy $S_{n}(t)$ for one dimensional harmonic oscillators with short-range couplings. This kind of springlike coupling corresponds to the kernel matrix $K=$ tridiag $\left\{-1,2+m^{2},-1\right\}$ in Eq. (1), where it has nonzero elements only in the main diagonal and the first diagonals below and above the main one. The parameter $m$ plays the role of the mass of the field theory. This kind of spring-like coupling can be considered as the discretization of a massive bosonic continuum theory given by the Klein-Gordon hamiltonian. In the continuum limit the Eq. (11) for the short-range harmonic oscillators has the following form:

$$
\mathcal{H}=\frac{1}{2} \int d x\left[\left(\partial_{t} \phi\right)^{2}+(\nabla \phi)^{2}+m^{2} \phi^{2}\right] .
$$

To determine the time evolution of the the von Neumann entropy $S_{A}(t)$ and the Rényi entropy $S_{n}(t)$ for the short range harmonic oscillators we used Eqs. (20) and (22). In this respect, we follow the method explained in the last section. Let us first discuss different configurations for the system and also subsystem that we have used in our study. We consider two different configurations of the system depicted in Fig. (11) among which we will calculate the entanglement:

$\mathfrak{g}_{1}$ : System is very large and $A$ is a small sub-system with length $l$.

$\mathfrak{g}_{2}$ : System is a semi-infinite line, $[0, \infty)$, and the subsystem $A$ is the finite interval $[0, l)$.

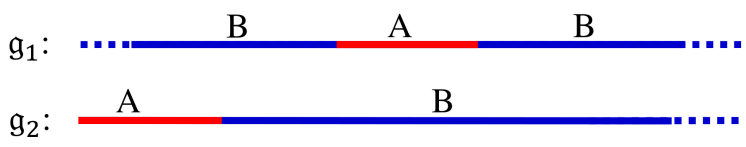

FIG. 1: (Color online) Different configurations of systems and subsystems for entanglement dynamics with global quenches.

Let us now focus on the case where the subsystem is very small with length $l$ and the system size $N$ is very large $\left(\mathfrak{g}_{1}\right)$. For this case we have used periodic boundary conditions so that $K(r)=K(r+N)$.

We are going to study the entanglement evolution after quantum quench. These are situations in which the system is initially prepared in the ground state of a hamiltonian of a gapped field theory $\left(m_{0} \neq 0\right.$ in Eq. 23). At $t=0$, the hamiltonian suddenly becomes massless, and the system is then allowed to evolve undisturbed for $t>0$. After the quench there is an energy excess in comparison with the ground state of the final hamiltonian $H$, which appear as quasiparticles and propagate to the entire system in time [6]. One of the interesting feature of the entanglement dynamics is the behavior for very short time. In this limit, the evolution of entanglement entropy is quadratic growth in time [31, 36] and follows

$$
S_{A}\left(t \ll t^{*}\right) \equiv \kappa_{2} t^{2},
$$

where $\kappa_{2}$ is a function of the mass parameter $m_{0}$.

After that, conformal field theory predicts the following formula for the dynamics of entanglement entropy [6]:

$$
S_{A}(t)=-\frac{c}{3} \log m_{0}+\left\{\begin{array}{ll}
\frac{\pi m_{0} c}{6} t & t<t^{*} \\
\frac{\pi m_{0} c}{12} l & t>t^{*}
\end{array},\right.
$$

where $m_{0}$ is the mass gap in the initial state and $t^{*}=l / 2$ is the saturation time. This behavior has a simple explanation in terms of quasiparticles excitations [6]. The initially excited state acts as a source for quasiparticle excitations. Highly entangled quasiparticle pairs created at a given point in space, travel with their maximum expected velocity $v$. A quasiparticle of velocity $v$ produced at $x$ is therefore at $x+v t$ at time $t$. Now consider a pair of quasiparticles emitted at $t=0$ from the midpoint of the subsystem $A$ ( $A$ is an interval of length $l$ ) and travel with $v=1$. They arrive simultaneously at a point $x^{\prime} \in A$ and $x^{\prime \prime} \in B$ at the same time $t=l / 2[\underline{6}]$. So the entanglement entropy between $x$ and $x^{\prime}$, saturates if $t>l / 2$. It is worth mentioning that quasiparticles emitted from far separated points (with the distance much larger than the correlation length $\xi=m_{0}^{-1}$ ) are incoherent.

The dynamics of Rényi entropies is given by the following formula:

$$
S_{n}(t)=-\frac{c_{n}}{3} \log m_{0}+\left\{\begin{array}{ll}
\frac{\pi m_{0} c_{n}}{6} t & t<t^{*} \\
\frac{\pi m_{0} c_{n}^{\prime}}{12} l & t>t^{*}
\end{array},\right.
$$




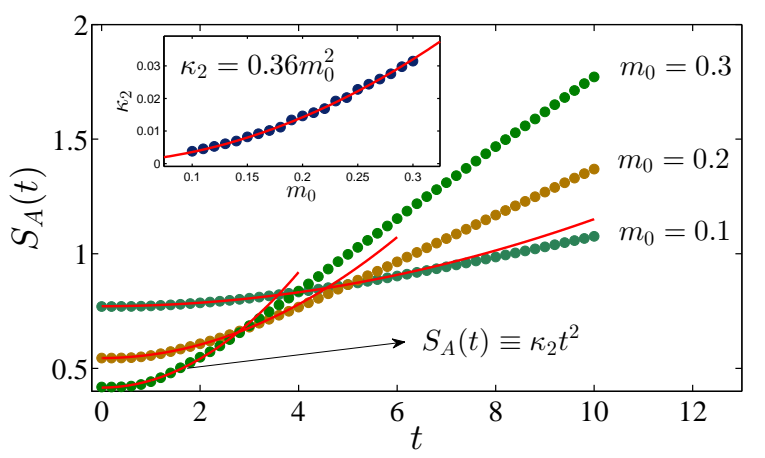

FIG. 2: (Color online) Quadratic growth in time for $S_{A}(t)$ in the region $t \ll t^{*}$ for the short-range harmonic oscillators with the configuration $\mathfrak{g}_{1}$. The total size of the system is $N=500$, and the subsystem size $l=60$. The solid red line corresponds to the $S_{A}(t) \equiv \kappa_{2} t^{2}$. Inset: The value of the scaling parameter $\kappa_{2}$ as a function of mass parameter $m_{0}$.

where $c_{n}=c_{n}^{\prime}=\frac{c}{2}(1+1 / n)$ and $c=1$.

Now we numerically evaluate the dynamics of von Neumann and Rényi entropies for the case $\mathfrak{g}_{1}$, where the system is a chain of $N$ coupled harmonic oscillators, and the subsystem is a very small region with length $l \ll N$ (see Fig. (11)). Here we consider a one-dimensional system of $N$ bosonic oscillators and we will measure the eigenvalues of the matrix $\mathcal{E}$ numerically. In order to calculate $\xi$ and $\theta$, we first need to construct the matrices $\Gamma$ and $K$. The matrix $K$ is the hamiltonian matrix after quench and the matrix $\Gamma=K_{0} \frac{1}{2}$ corresponds to the hamiltonian matrix which is prepared at time $t=0$. The matrix $A(t)$ can then easily be calculated from Eq. (7). Having the matrices $A(t)$ and $A^{-1}(t)$ we can now calculate the matrix $\mathcal{E}=-2(\tilde{\mathcal{C}})^{-1} \mathcal{D}$ from $\mathcal{C}$ and $\mathcal{D}$ (see Eq. (10)). The numerical results are summarized in the following.

Let us first address the behavior of the von Neumann entropy at the very short time $t \ll l / 2$. Our results show that $S_{A}(t)$ quadratically depends on $t$. The von Neumann entropy $S_{A}\left(t \ll t^{*}\right)$ for different values of $m_{0}$ is plotted in Fig. (2). The same behavior already noticed in Ref. [36]. In Fig. (2) we also present the result for the coefficient $\kappa_{2}$ as a function of mass parameter $m_{0}$ that is quadratic in $m_{0}$. Then until the saturation time $t^{*} \sim l / 2$, entanglement dynamics $S_{A}(t)$ grows linearly with time $\left(S_{A}(t) \sim \kappa_{1} t\right)$ and finally it saturates to the $S_{\infty}$ (see Fig. (3)-a). The coefficient $\kappa_{1}$, is a linear function of mass parameter $m_{0}$ (see Fig. (3)-b) with the same slop as the predicted value $\pi / 6$. We also obtained the behavior of the saturation value $S_{\infty}$ with respect to the mass term $m_{0}$. In Fig. (3-c) we show numerical results for the linear dependence between $S_{\infty}$ and $m_{0}$ which the slope matches perfectly with the theoretical value $\pi l / 12$. One can do the same calculation for different values of $l$ which the result will be the same. In Fig. (3-d) we also sketched $S_{A}(t)$ versus $t$ for the fix value of $m_{0}=0.4$ and different values of $l$. The entanglement entropy for such a system gets saturated in which $S_{\infty}$ increases lin-
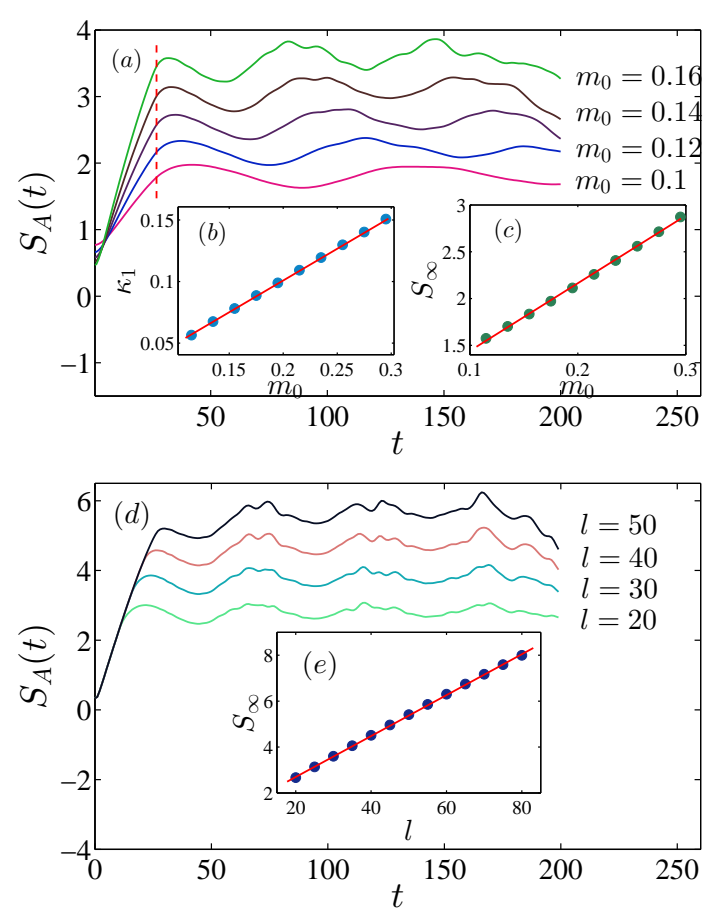

FIG. 3: (Color online) Top: (a) Entanglement entropy dynamics $S_{A}(t)$ for short-range harmonic oscillator with the configuration $\mathfrak{g}_{1}$ and different mass parameter $m_{0}$. The total size of the system is $N=400$, and the subsystem size $l=50$. The dashed line corresponds to the saturation time $t^{*}=l / 2$. (b) The von Neumann entropy $S_{A}(t)$ in the $t<l / 2$, limit obeys the $S_{A}(t) \sim \kappa_{1} t$, where $\kappa_{1}$ is a linear function of mass $m_{0}$. (c) In the $t>l / 2$ limit $S_{A}(t)$ saturates to the $S_{\infty}$. The value of $S_{\infty}$ for the fixed value of $l=30$ is linear function of $m_{0}$. Bottom: (d)The von Neumann entropy $S_{A}(t)$ for short range harmonic oscillators with the configuration $\mathfrak{g}_{1}$ and fixed value of the mass parameter $m_{0}=0.4$. The total size of the system is $N=400$, and the subsystem size $l \in\{20,30,40,50\}$. (e) In the $t>l / 2$ limit, $S(t)$ saturates to the $S_{\infty}$. The value of $S_{\infty}$ is a linear function of $l$.

early with $l$ (see Fig. (3)-e). A good agreement has been found between our numerical results and the theoretical prediction $\pi m_{0} / 12$.

Using Eq. (22), we can also evaluate the time evolution of Rényi entropy $S_{n}(t)$. In Fig. (4) we have plotted the numerical estimates of the scaling factors $c_{n}$ and $c_{n}^{\prime}$. The agreement between the numerical results and the theoretical prediction Eq. (26) is fairly good.

The next step is to analyze the dynamics of von Neumann and Rényi entropies for the case $\mathfrak{g}_{2}$, where the system is semi-infinite line, and the subsystem is finite interval $[0, l$ ) (see Fig. 1). It is clear that to understand the qualitative behavior of entanglement dynamics, a very similar explanation that is based on quasi-particle picture, should apply also for this special case of system and subsystem configuration. The only difference here is that the quasiparticles emitted from $x=0$ in the region $A$ need more time to be observed by the first point $x=l$ in the region $B$. Therefore one can expect the saturation 


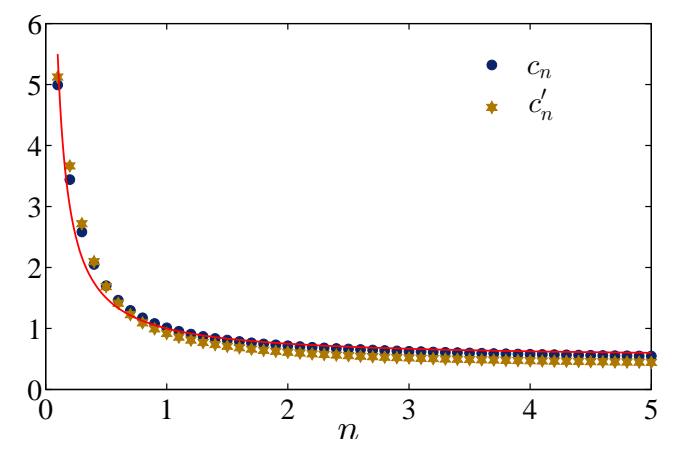

FIG. 4: (Color online) Prefactors $c_{n}$ and $c_{n}^{\prime}$ for dynamics of Rényi entropy for the short range harmonic oscillator system with configuration $\mathfrak{g}_{1}$ and the system size $N=500$. The solid red line represents $\frac{1}{2}(1+1 / n)$ (see Eq. (27)).

time is $t^{*}=l$. In this case we have the following formula

$$
S_{n}(t)=-\frac{c_{n}}{6} \log m_{0}+\left\{\begin{array}{ll}
\frac{\pi m_{0} c_{n}}{12} t & t<t^{*} \\
\frac{\pi m_{0} c_{n}^{\prime}}{12} l & t>t^{*}
\end{array},\right.
$$

where $c_{n}=c_{n}^{\prime}=\frac{1}{2}(1+1 / n)$.

To determine the time evolution of the von Neumann entropy for short-range harmonic oscillator with configuration $\mathfrak{g}_{2}$, we computed the eigenvalues of the matrix $\mathcal{E}$ to evaluate $S_{A}$ and $S_{n}$ by Eqs. (20) and (22). In Fig. (5) we show $S_{A}(t)$ for different values of $m_{0}$ and $l$ respectively. Our numerical results show that $S_{A}(t)$ in very short time is quadratic in time. Then the evolution of $S_{A}(t)$ has been studied for intermediate times that is $S_{A}(t)=\kappa_{1} t$. The prefactor $\kappa_{1}$ as a linear function of $m_{0}$ is depicted in Fig. (5-b). We also calculated the prefactor of the $\kappa_{1}$ with respect to $m_{0}$. There is a very good agreement with the predicted value $\pi / 12$.

Next we examine the scaling properties of the entanglement entropy $S_{\infty}$ in saturation regime. Figures (5-c) and (5-e) show a linear plot of $S_{\infty}$ versus of $m_{0}$ and $l$. The straight lines, with slopes $\pi l / 12$ and $\pi m_{0} / 12$, respectively, are drown to demonstrate the good agreement with the prediction Eq. (27). Finally we characterized the time evolution of the Rényi entropy and our numerical observation was in perfect agreement with the Eq. (27).

In the next section, our main results on the time evolution of the entanglement entropy for long-range harmonic oscillator are presented. To avoid any finite size effect we will concentrate on the case where the system is very large and the subsystem has small size $l$ (case $\mathfrak{g}_{1}$ in Fig. 1).

\section{HARMONIC SYSTEMS WITH LONG-RANGE COUPLINGS}

In this section, we study the time evolution of the entanglement after global quench for the system of har-
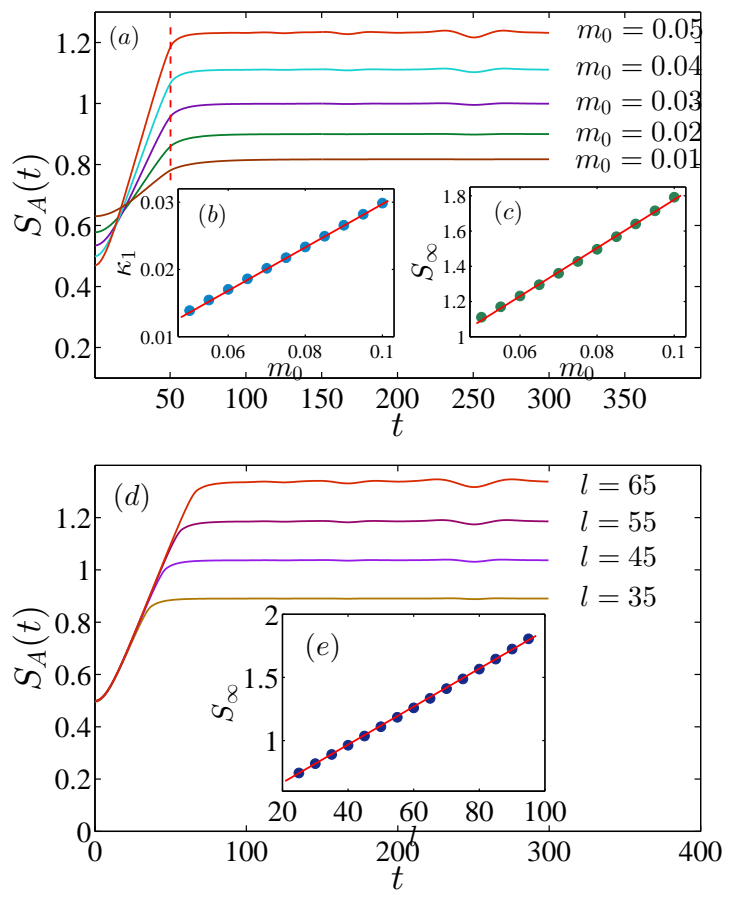

FIG. 5: (Color online) Top: (a) Entanglement entropy dynamics $S_{A}(t)$ for short-range harmonic oscillator with the configuration $\mathfrak{g}_{2}$ and different mass parameter $m_{0}$. The total size of the system is $N=500$, and the subsystem size $l=50$. The dashed line corresponds to the saturation time $t^{*}=l$. (b) $S_{A}(t)$ in the $t<l$, limit obeys the $S(t)=\kappa_{1} t$, where $\kappa_{1}$ is a linear function of mass $m_{0}$. (c) $S_{A}(t)$ in the $t>l$ limit saturates to the $S_{\infty}$. The value of $S_{\infty}$ is linear function of $m_{0}$. Bottom: (d) Entanglement entropy dynamics $S_{A}(t)$ for short range harmonic oscillators with the configuration $\mathfrak{g}_{2}$ and fixed value of the mass parameter $m_{0}=0.05$. The total size of the system is $N=500$, and the subsystem size $l \in\{35,45,55,65\}$. (e) The value of $S_{A}(t)$ in the $t>l$ limit saturates to the $S_{\infty}$ which is a linear function of $l$.

monic oscillators with long-range couplings. Following Eq. (11), let us introduce the $K$ matrix for coupled harmonic oscillators with long-range couplings. There are many ways to write a long range $K$ matrix for nonlocal scalar field theories $i$. e. see Ref. [26], however, we are interested in those that are defined by the fractional laplacian operator. In the continuum limit the fractional laplacian has simple Fourier transform $|q|^{\alpha}$ or $\left(q^{2}\right)^{\frac{\alpha}{2}}$ which $q^{2}$ is just the Fourier transform of a simple laplacian. Since the discrete laplacian in the fourier representation is $2-2 \cos q$, so that one may use the appropriate power of this to define the discrete fractional laplacian. Then the elements of the interaction kernel $K$, representing the discretized fractional laplacian are then given by

$$
\begin{aligned}
K_{i, j}= & -\int_{0}^{2 \pi} \frac{d q}{2 \pi} e^{i q(i-j)}\left\{[2(1-\cos (q))]^{\frac{\alpha}{2}}+m^{\alpha}\right\} \\
& =\frac{\Gamma\left(-\frac{\alpha}{2}+i-j\right) \Gamma(\alpha+1)}{\pi \Gamma\left(1+\frac{\alpha}{2}+i-j\right)} \sin \left(\frac{\alpha}{2} \pi\right)+m^{\alpha} \delta_{i, j}
\end{aligned}
$$


The above equation for large distances behaves like $K_{i, j} \sim 1 /|i-j|^{1+\alpha}$. This power-law interaction for $\alpha \geq 2$ is effectively local. In the continuum limit the Eq. (28) can be written as

$$
\frac{1}{2} \sum_{i, j=1}^{N} \phi_{i} K_{i j} \phi_{j} \rightarrow \int\left[-\frac{1}{2} \phi(x)(-\nabla)^{\alpha / 2} \phi(x)+\frac{1}{2} m^{\alpha} \phi^{2}\right] d x .
$$

In the above formula $(-\nabla)^{\alpha / 2}$ corresponds to the fractional laplacian operator.

The most important characteristics of the oscillator system is the spatial correlation $\left\langle\phi_{l} \phi_{m}\right\rangle$, where for the system with periodic boundary condition, one can find the spatial correlation length $\xi_{s}$ as:

$$
\begin{aligned}
\xi_{s}^{-1} & \equiv-\lim _{r \rightarrow \infty} \frac{1}{r} \log \left|\left\langle\phi_{l} \phi_{l+r}\right\rangle\right| \\
& =-\lim _{r \rightarrow \infty} \frac{1}{r} \log \left|K^{-1 / 2}(r)\right| .
\end{aligned}
$$

The elements of $K^{-1 / 2}$ for a sufficiently large onedimensional system can be expressed as a Fourier series

$$
\begin{aligned}
& K^{-1 / 2}(r, m)= \\
& -\int_{0}^{2 \pi} \frac{d q}{2 \pi} e^{i q r} \quad\left\{[2(1-\cos (q))]^{\frac{\alpha}{2}}+m^{\alpha}\right\}^{-1 / 2} .
\end{aligned}
$$

In the scaling limit Eq. (31) behaves like $K^{-1 / 2}=$ $-\int d q e^{i q r}\left\{|q|^{\alpha}+m^{\alpha}\right\}^{-1 / 2}$ and one can evaluate this integral for $m=0$ which is given by:

$$
K^{-1 / 2}(r, 0) \sim 1 / r^{1-\alpha / 2} .
$$

We now want to evaluate the integral Eq. (31) for the massive case $m \neq 0$ and large values of the distanse $r$. The integral can not be done explicitly in the most general case, therefore we studied this case just numerically (see Appendix. (A). We find that the best fit to our numerical data is:

$$
K^{-1 / 2}(r, m) \propto 1 / r^{1+\alpha} .
$$

One can observe that the above result leads to $\xi_{s}^{-1}=0$ for all values of the mass parameter $m$. Note that for the special case $\alpha=2$, correlation function decays exponentially and the correlation length is proportional to $\xi_{s} \sim 1 / m$. It is useful to note that $\alpha>2$ corresponds to a a system of harmonic oscillators with long-range $(\alpha<2)$ plus shot-range $(\alpha=2)$ interactions where we have $\alpha=2$ and $\alpha<2$ both laplacians in the hamiltonian. Therefore, all the massive correlations are exponential because everything is dominated with $\alpha=2$.

\section{A. Weakly coupled long-range harmonic oscillators $1<\alpha<2$}

To understand the entanglement entropy growth behavior for harmonic oscillators with weak couplings we consider the system with very large size and a subsystem

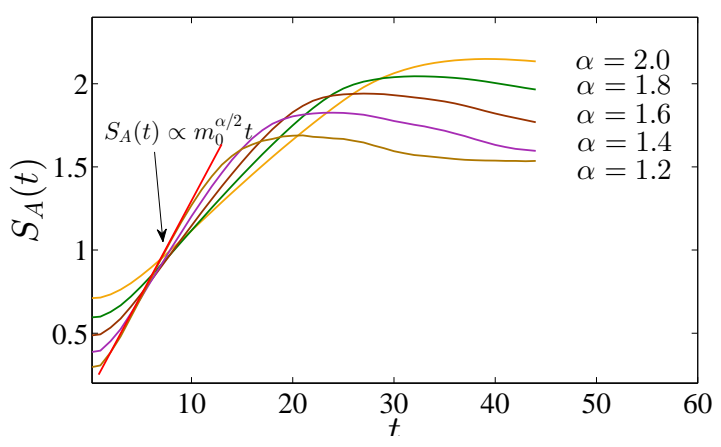

FIG. 6: (Color online) Entanglement entropy dynamics $S_{A}(t)$ in the long-range harmonic oscillators with the configuration $\mathfrak{g}_{1}$ for different values of $\alpha$. The curves correspond to the linear change of the $S_{A}(t)$ with time $t$ before the saturation time $t^{*}$ for the $1<\alpha \leq 2$. The total size of the system is $N=400$, the mass parameter $m_{0}=0.1$ and the subsystem size $l=50$.

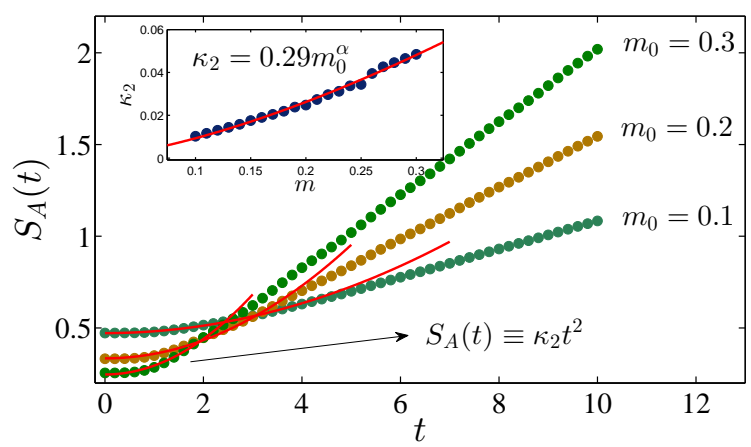

FIG. 7: (Color online) Quadratic growth in time for $S_{A}(t)$ where $t \ll t^{*}$. The harmonic system is long-range $(\alpha=1.5)$ with the configuration $\mathfrak{g}_{1}$. The total size of the system is $N=500$, and the subsystem size $l=60$. The solid red line corresponds to the $S_{A}(t) \equiv \kappa_{2} t^{2}$. Inset: The value of the scaling parameter $\kappa_{2}$ as a function of mass parameter $m_{0}$.

with length $l$ (configuration $\mathfrak{g}_{1}$ in the Fig. (10). First one should construct the interaction kernel $K$ from Eq. (28). The next step is to analyze the eigenvalues of the matrix $\mathcal{E}$ and measure the entanglement entropy $S_{A}(t)$ using Eq. (20). We display the resulting quantity for different values of $\alpha$, in Fig. (6).

An interesting behavior is the quadratic entropy growth at the very short-time $t \ll t^{*}$. In Fig. (7) we plot the entanglement entropy dynamics $S_{A}(t)$ shortly after the quench. This is the same qualitative quadratic behavior $S_{A} \equiv \kappa_{2} t^{2}$ as seen in the short range case that we mentioned in the Eq. (24). Our results indicate that the prefactor $\kappa_{2}$ obeys the power law formula $\kappa_{2} \sim m_{0}^{\alpha}$.

As we remarked before, if we consider the configuration $\mathfrak{g}_{1}$ for harmonic oscillators with $\alpha=2$, according to Eq. (27) we expect a linear growth of the entropy as a function of time. Notice that this linear growth is only true before saturation time $t^{*}$ and after that the $S_{A}(t)$ will become time independent. Figure (6) clearly shows 

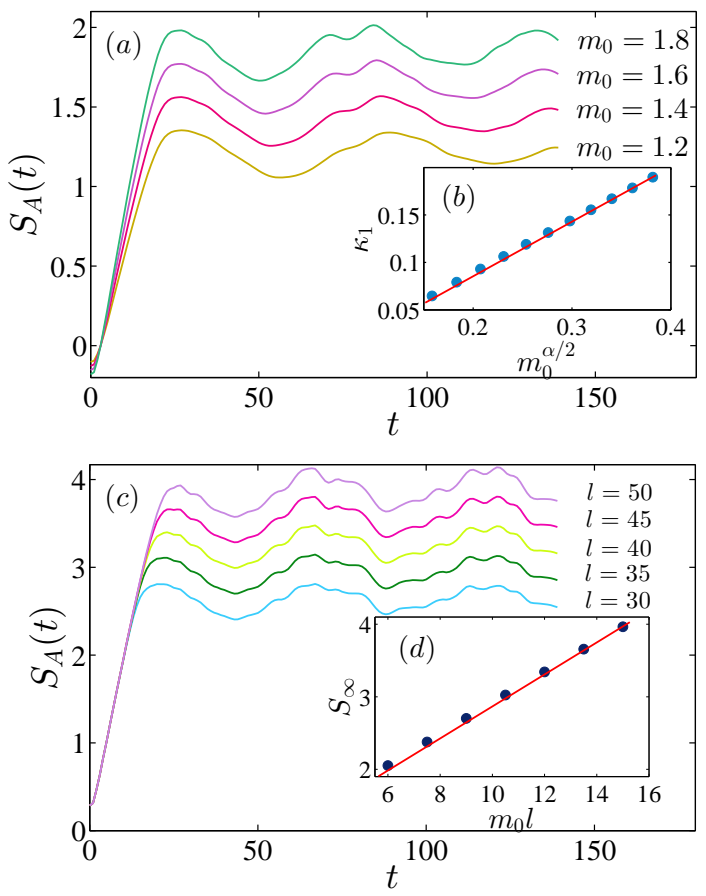

FIG. 8: (Color online) Top: (a) Entanglement entropy dynamics $S_{A}(t)$ for long-range harmonic oscillators $(\alpha=1.6)$ with the configuration $\mathfrak{g}_{1}$ and different mass parameter $m_{0}$. The total size of the system is $N=500$, and the subsystem size $l=50$. (b) Before the saturation time $t<t^{*}$, entanglement entropy dynamics obeys the $S(t) \sim \kappa_{1} t$, where $\kappa_{1}$ is a linear function of $m^{\alpha / 2}$. Bottom: (c) The entanglement entropy dynamics $S_{A}(t)$ for long-range harmonic oscillator with the configuration $\mathfrak{g}_{1}$ and fixed value of mass parameter $m_{0}=0.3$. The total size of the system is $N=300$, and the subsystem size $l \in\{30,35,40,45,50\}$. (d) $S_{A}(t)$ in $t \gg t^{*}$, saturates to the $S_{\infty}$ where it changes linearly with $m_{0} l$.

that $S_{A}(t)$ grows as $\sim t$ before saturation begins. We see that this linear form for the entanglement entropy is hold in all cases with $1<\alpha \leq 2$. Figure (8) is the plot of $S_{A}(t)$ for long-range harmonic oscillator with $\alpha=1.6$ and different values of the initial mass parameter $m_{0}$. Our numerical simulations show that the slope of linear entanglement growth in time is $\kappa_{1}=\mathcal{A} m_{0}^{\alpha / 2}$. In Fig. (8), we plot a comparison of our estimate with the numerical results. We also see that the prefactor $\mathcal{A}$ as we demonstrated in Fig (9) is $\alpha$-independent and it is equal to the value $\pi / 6$.

We will now ask how the entanglement dynamics $S_{A}(t)$ for system with long-range couplings depends on the subsystem size $l$. In Fig. (8) we show the numerical results for the time evolution of the entanglement dynamics in the long-range harmonic oscillators with $\alpha=1.6$ for different values of the subsystem size $l$. It is clear from Fig. (8) that at long time $S_{A}(t)$ saturates to $S_{\infty}$. We find that the saturation value, $S_{\infty}$, grows linearly as $S_{\infty}=\mathcal{B} m_{0} l$. As shown in the Fig. (91), the prefactor $\mathcal{B}$ is surprisingly $\alpha$-independent and equal to the value $\pi / 12$.

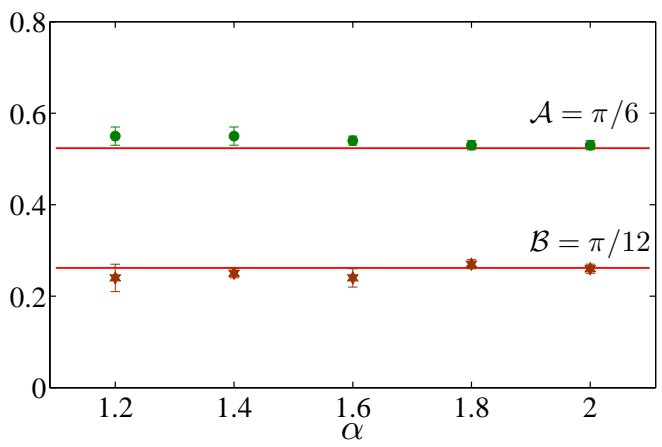

FIG. 9: (Color online) Numerical results for the coefficients $\mathcal{A}$ and $\mathcal{B}$ as a function of $\alpha$ (see Eq. (34)).

In summary, the time evolution of the entanglement entropy for long-range harmonic oscillators follows

$$
S_{A}(t)=-\frac{c^{g}(\alpha)}{3} \log m_{0}+\left\{\begin{array}{ll}
\mathcal{A} m_{0}^{\alpha / 2} t & t<t^{*} \\
\mathcal{B} m_{0} l & t \gg t^{*}
\end{array} .\right.
$$

where the prefactor $c^{g}(\alpha)$ is introduced in Refs. 22, 23]. In all cases with $1<\alpha<2$, we find that the prefactors $\mathcal{A}$ and $\mathcal{B}$, as shown in Fig. (9), are independent of $\alpha$.

It is instructive to compare the scaling parameters $\kappa_{2} \sim m_{0}^{\alpha}$ and $\kappa_{1} \sim m_{0}^{\alpha / 2}$ the respective coefficient of the quadratic and linear part of the $S_{A}(t)$. This gives us an insight into the special behavior of the entanglement dynamics of the harmonic systems with long-range couplings. As we will soon see, this behavior is closely related to the form of the hamiltonian Eq. (29).

Let us consider now the time evolution of the Rényi entropy for the harmonic system with long-range couplings. Figure (10) is devoted to these analysis. Interestingly, we observe that

$$
S_{n}(t)=-\frac{c_{n}^{g}(\alpha)}{3} \log m_{0}+\left\{\begin{array}{ll}
\mathcal{A}_{n} m_{0}^{\alpha / 2} t & t<t^{*} \\
\mathcal{B}_{n} m_{0} l & t \gg t^{*}
\end{array},\right.
$$

where the prefactor $c_{n}^{g}(\alpha)$ is introduced in Refs. 22, 23. The scaling parameters $\mathcal{A}_{n}$ and $\mathcal{B}_{n}$ are depicted in the Fig. (10) for different values of $\alpha$. It is surprising that $6 \mathcal{A}_{n} / \pi$ and $12 \mathcal{B}_{n} / \pi$ are $\alpha$-independent and follow the relation $\frac{1}{2}(1+1 / n)$. The specific reason for this $\alpha$ independent behavior remains an open problem.

Since we were able to calculate the entanglement entropy dynamics $S_{A}(t)$, we can now numerically evaluate another aspect to consider which is the saturation time $t^{*}$. It is easy to see that (see Fig. (6) ) the entanglement entropy saturates at time $t=t^{*}$. We measured this value from our numerical data. It is clear from Fig. (11) that $t^{*}$ scales with the subsystem size as

$$
t^{*}=\lambda(l / 2)^{\alpha / 2} .
$$




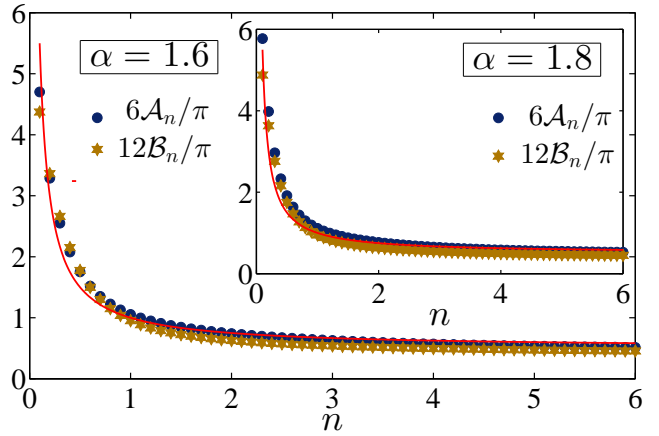

FIG. 10: (Color online) Scaling prefactors $\mathcal{A}_{n}$ and $\mathcal{B}_{n}$ vs $n$ for different $\alpha$ 's $(\alpha=1.6,1.8)$. The solid red line represents $\frac{1}{2}(1+1 / n)$.

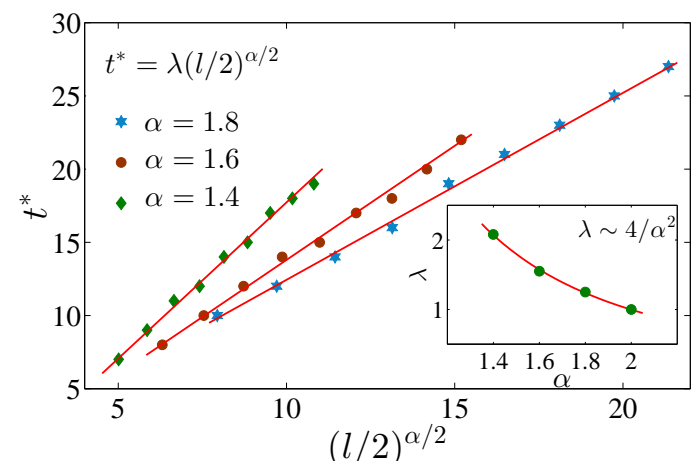

FIG. 11: (Color online) (a) The saturation time $t^{*}$ versus $(l / 2)^{\alpha / 2}$ for different values of $\alpha$. (b) Scaling parameter $\lambda$ with respect to $\alpha$.

The prefactor $\lambda$ is $\alpha$-dependent quantity where the best fitting function to our numerical data is $\lambda=4 / \alpha^{2}$. It is worth mentioning that this finding nicely matches the special case $t^{*}=l / 2$ for those harmonic systems with short range couplings.

Let us now discuss an important consequence regarding the above results. The differences in the behavior of the entanglement entropy dynamics for short and longrange harmonic oscillators come from the difference between length and time scales. It is well known that in the long-range systems the dynamical exponent is $z=\alpha / 2$. The dynamical exponent controls the relative scaling of time and space leading to the invariant form $t / l^{z}$. Therefore this argument hints that for the massive system there are two correlation lengths $\xi_{t}=1 / m^{z}$ and $\xi_{s}=1 / m$ where they will be equal for harmonic system with shortrange couplings $(\alpha=2)$. Returning to our entanglement dynamics problem, we can conclude that the saturation time $t^{*}$ should scale as Eq. (36). In a similar way one can guess the behavior in the Eq. (34).

\section{B. Strongly coupled long-range harmonic oscillators $0<\alpha<1$}

So far, we have only considered long-range harmonic chains with $1<\alpha \leq 2$. Here, we explore entanglement dynamics after a quantum quench in long-range harmonic oscillators with strong couplings $(\alpha<1)$. We observe that, $S_{A}(t)$ does not grow linearly and also it does not saturate in the finite time interval that we considered. It is interesting to note that the entanglement dynamics for this case has the form,

$$
S_{A}(t)=-\frac{c^{g}(\alpha)}{3} \log m_{0}+ \begin{cases}\kappa_{2} t^{2} & t \ll 1 \\ \mathcal{P}\left(m_{0}, l, \alpha\right) \log t & \\ \mathcal{B} m_{0} l & t \gg 1\end{cases}
$$

where $c^{g}(\alpha)$ is introduced in Refs. [22, 23], and $\kappa_{2} \propto m_{0}^{\alpha}$. In our numerical analysis, we found that, $\mathcal{B} \sim \pi / 12$ is independent of $\alpha$. In Fig. (12) we report the logarithmic behavior of the entanglement entropy dynamics for the strongly long range harmonic oscillator. As we show in Fig. (12), $\mathcal{P}\left(m_{0}, l, \alpha\right)$ is a function of mass parameter $m_{0}$ and size of the sub-system $l$, as

$$
\mathcal{P}\left(m_{0}, l, \alpha\right)=\left[\mathcal{V}_{1}(\alpha)\left(m_{0} l\right)^{\alpha / 2}+\mathcal{V}_{2}(\alpha)\right],
$$

where $\mathcal{V}_{1}$ and $\mathcal{V}_{2}$ as shown in the Fig. (12) are functions of $\alpha$. In Fig. (13) we depicted the entanglement dynamics for strongly long-range harmonic oscillators in the saturation regime. In this regime the entanglement entropy for all values of $\alpha$ saturates to the $S_{\infty} \propto m_{0} l$ (see Eq. (37)).

As pointed out above, in this study we considered a system of long-range harmonic oscillators with the interaction kernel Eq. (28). To study the time evolution of von Neumann and Rényi entropies after quantum quench we assume that the mass parameter in the massive hamiltonian Eq. (28) is suddenly changes from $m_{0}$ to a different value $m \sim 0$. Hence after the quench there is an energy excess which acts as a source of quasiparticles moving with maximum group velocity. The maximum group velocity for $1<\alpha<2$ generally depends on the mass parameter $m$ as

$$
v_{g}^{\max } \propto m^{\alpha / 2-1},
$$

which obviously diverges in the limit $m \rightarrow 0$ (see Appendix. (B). Therefore, for those long-range systems that have been quenched to the critical point of the system $(m=0)$, there is no maximum group velocity for quasiparticles. As already reported in the previous sections, a very small mass parameter $\left(m \ll m_{0}\right)$ has been chosen to avoid rapid oscillations of $S_{A}(t)$ in saturation regime. It is worth mentioning that our results have not been affected by non-zero parameter $m$ until $m \gg 1 / N$ which $N$ 


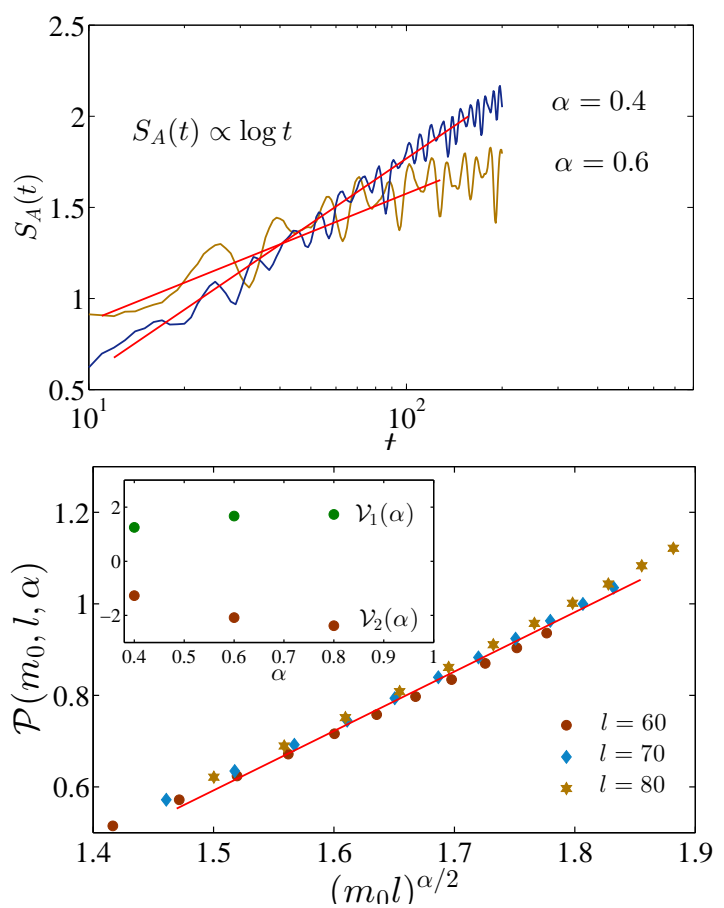

FIG. 12: (Color online) Top: Entanglement entropy dynamics $S_{A}(t)$ in long-range harmonic oscillators $(\alpha<1)$ with the configuration $\mathfrak{g}_{1}$. The total size of the system is $N=500$, the mass parameter $m_{0}=0.1$ and the subsystem size $l=50$. The red lines corresponds to logarithmic change of $S_{A}(t) \sim \mathcal{P}\left(m_{0}, l, \alpha\right) \log t$ where the coefficient $\mathcal{P}\left(m_{0}, l, \alpha\right)$ is a function of $m_{0}, l$ and $\alpha$. Bottom: The coefficient $\mathcal{P}\left(m_{0}, l, \alpha\right)$ for different values of $m_{0}$ and $l$ is a linear function of $\left(m_{0} l\right)^{\alpha / 2}$, i.e. $\mathcal{P}\left(m_{0}, l, \alpha\right)=\mathcal{V}_{1}(\alpha)\left(m_{0} l\right)^{\alpha / 2}+\mathcal{V}_{1}(\alpha)$ where the coefficients $\mathcal{V}_{1}\left(\mathcal{V}_{2}\right)$ as shown in the inset, is an increasing (decreasing) function of $\alpha$.

is the size of the system. In the Appendix we explain all of the above in details. This means that interestingly we found the linear behavior for the time evolution of von Neumann entropy $S_{A}(t)$ even for those systems with infinite maximum group velocity. To compare such result with the theoretical predictions for free field theory, we have taken into account that maximum group velocity for short-range harmonic oscillator is finite.

Following Ref. 44 it has been shown that the time evolution of the entanglement entropy in the transverse field Ising model with long-range interactions $\left(J_{i, j} \propto\right.$ $|i-j|^{-\sigma}$ ) shows three different regimes as a function of $\sigma$. For relatively short-range interactions $\sigma \geq 1$ a linear growth of the entanglement entropy as a function of time has been found. For those long-range interactions with $\sigma \in(0.8,1.0)$ a regime of logarithmic growth of entropy has been proposed. Finally for strongly long-range interactions with $\sigma<0.2$ rapid oscillations of the entanglement entropy around small values are found. In light of our entanglement dynamics results in the harmonic oscillators with long-range couplings, similar regimes possibly with similar signatures, as in, Ref. [44] have been found.
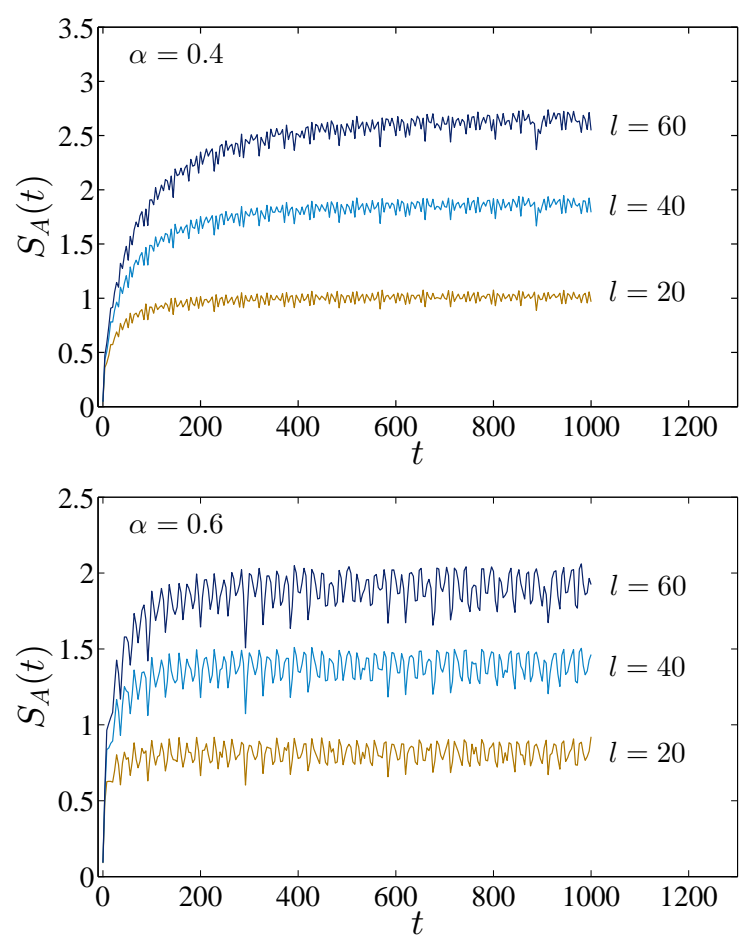

FIG. 13: (Color online) The role of finite size effects on the entanglement entropy dynamics $S_{A}(t)$ in long-range harmonic oscillators $(\alpha<1)$ with the configuration $\mathfrak{g}_{1}$. The total size of the system is $N=500$, the mass parameter $m_{0}=0.1$. Top: $\alpha=0.4$, Bottom: $\alpha=0.6$.

Note that in this study we have $\sigma=1+\alpha$. Our findings show that the linear growth of entanglement entropy breaks down at $\alpha=1(\sigma=2)$. The behavior of $S_{A}(t)$ for $\alpha>1(\sigma>2)$ is a linear function of time and on the other hand it follows the logarithmic growth with time for $\alpha<1(\sigma<2)$. In principle following [51] one should be able to find some useful linear bounds on the growth of entanglement entropy for all the values of $\alpha$. However, it is not clear that why in our system for $\alpha>1$ the bound is saturated and for $\alpha<1$ it is not.

\section{INITIAL STATE EFFECTS}

In the previous sections, we studied the time evolution of the entanglement entropy after global quantum quench. We have considered the harmonic oscillator systems with short and long-range couplings. The most crucial point about the long-range hamiltonian Eq. (28) is that the gapfull initial state has the power law correlation function (see Eq. (33)). One of the fundamental questions that has to be addressed is how the entanglement dynamics is related to the initial state of the system. Here, we would like to study the time evolution of entanglement entropy for the long-range systems with the gapless power-law, exponential decaying and unentangled initial states. 

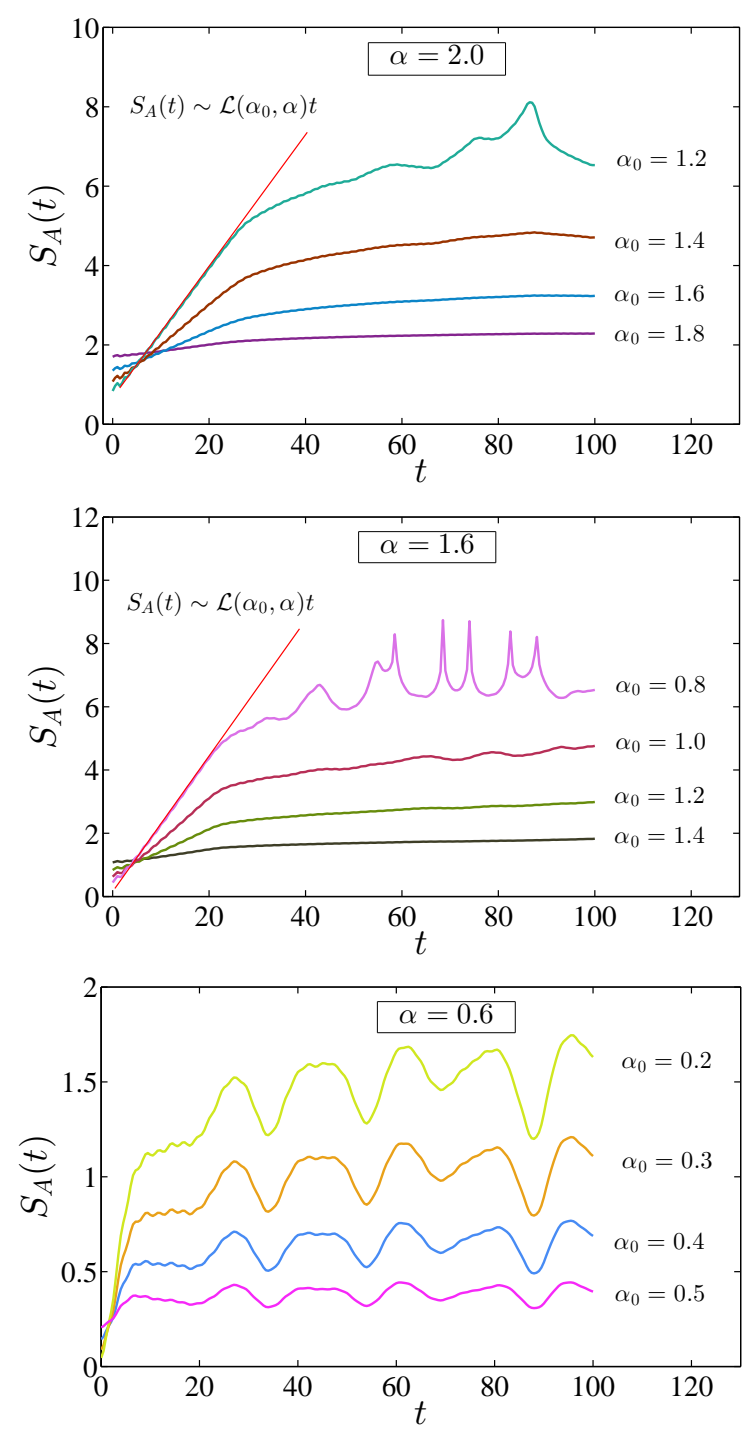

FIG. 14: (Color online) Top: The time evolution of the von Neumann entropy $S_{A}(t)$ in the short-range harmonic oscillators with the total size $N=400$ when the initial state is gapless power-law with long-range scaling exponent $\alpha_{0}$. $S_{A}(t)$ starts from non-zero value and grows linearly with time then saturates. Middle: The time evolution of the von Neumann entropy $S_{A}(t)$ in the harmonic oscillators with weak long-range coupling $(\alpha=1.6)$. Bottom: The same result for the harmonic oscillators with strong long-range couplings $(\alpha=0.6)$.

\section{A. Gapless power-law initial state}

To extend our analysis to gapless initial state with power-law correlation function, we first need to modify the previous definition of the global quench. As mentioned earlier, for the gapless $(m=0)$ long-range harmonic oscillators given by Eq. (29), the correlation function is of the form $K^{-1 / 2}(r) \propto 1 / r^{1-\alpha / 2}$ (see Sec. (V) ). Let us now consider the quench between gapless power law initial state with the scaling exponent $\alpha_{0}$ to another gapless long-range hamiltonian with the scaling factor $\alpha$. We are mainly interested in the general time and subsystem size dependence of the entanglement entropy in the case $\alpha_{0} \neq \alpha$. Here we assume that $\alpha_{0}<\alpha$. Shown in Fig. (14) are plots of the time evolution of the von-Neumann entanglement entropy for various values of $\alpha_{0}$ and $\alpha$. We observe that the von-Neumann and Rényi entropies for the different values of the $\alpha_{0}$ and $\alpha$ grow linearly in time, then saturate at long time. We see that the numerical data are fit very well by the following formula for the Rényi entropi follows

$$
S_{n}(t)=\frac{\tilde{c}_{n}\left(\alpha_{0}\right)}{3} \log l+ \begin{cases}\mathcal{L}_{n}\left(\alpha_{0}, \alpha\right) t & t<t^{*} \\ \mathcal{L}_{n}\left(\alpha_{0}, \alpha\right) b(\alpha) l & t>t^{*}\end{cases}
$$

where $l$ is the size of the subsystem and the prefactor $\tilde{c}_{n}\left(\alpha_{0}\right)$ is introduced in Ref. [23] and $t^{*}=b(\alpha) l$. Since the coefficient $\mathcal{L}_{n}\left(\alpha_{0}, \alpha\right)$ only depends on $n$ and the initial and final scaling parameters $\alpha_{0}$ and $\alpha$, we obtain the asymptotic relation

$$
\mathcal{L}_{n}\left(\alpha_{0}, \alpha\right)=\mathcal{J}_{n} \ln \left(\alpha / \alpha_{0}\right),
$$

where our numerical results suggest that $\mathcal{J}_{n}=\frac{1}{6}(1+1 / n)$. In Fig. (15) the prefactor $\mathcal{L}\left(\alpha_{0}, \alpha\right)$ (for $n=1$ ) as a function of $\alpha_{0}$ and $\alpha$ is reported. The numerical results for the prefactor $\mathcal{J}_{n}$ is shown in the bottom panel of Fig. (15), pointing at a good agreement between $\mathcal{J}_{n}=$ $\frac{1}{6}(1+1 / n)$ and our numerical results.

We should note that our numerical calculations show that for the fixed values of the scaling parameters $\alpha_{0}$ and $\alpha$, the saturation regime begins earlier for smaller value of the subsystem size $l$. In Fig. (16) we show numerical results for the saturation time $t^{*}$ as function of the subsystem size $l$ for different values of $\alpha$ (the results are $\alpha_{0}$ independent). The figure shows that $t^{*}$ is a linear function of $l$ as $t^{*}=b(\alpha) l$. As mentioned previously, the saturation time for the gapfull power-law initial state scales like Eq. (36). The specific reason for the linear behavior in the case of gapless power-law initial state remains unsolved. The parameter $b(\alpha)$ is shown in the Fig. (16) and best fit to our numerical data was $b(\alpha) \simeq$ $0.4 \alpha-0.3$. The value of $b(\alpha)$ is zero at $\alpha \sim 0.75$. It is interesting to note that for those long-range harmonic oscillators with strong long-range couplings $(\alpha<1.0)$ the typical behavior of the entanglement entropy changes qualitatively (see Fig. (144)). In other words, there is no linear behavior in the entanglement growth.

\section{B. Exponential decaying initial state}

In order to analyze the initial state effects, we define the hamiltonian of the long-range harmonic oscillator with the following $K$ matrix:

$$
K_{i, j}=\int_{0}^{2 \pi} \frac{d q}{2 \pi} e^{i q(i-j)}\left[(2-2 \cos (q))+m^{2}\right]^{\frac{\alpha}{2}},
$$



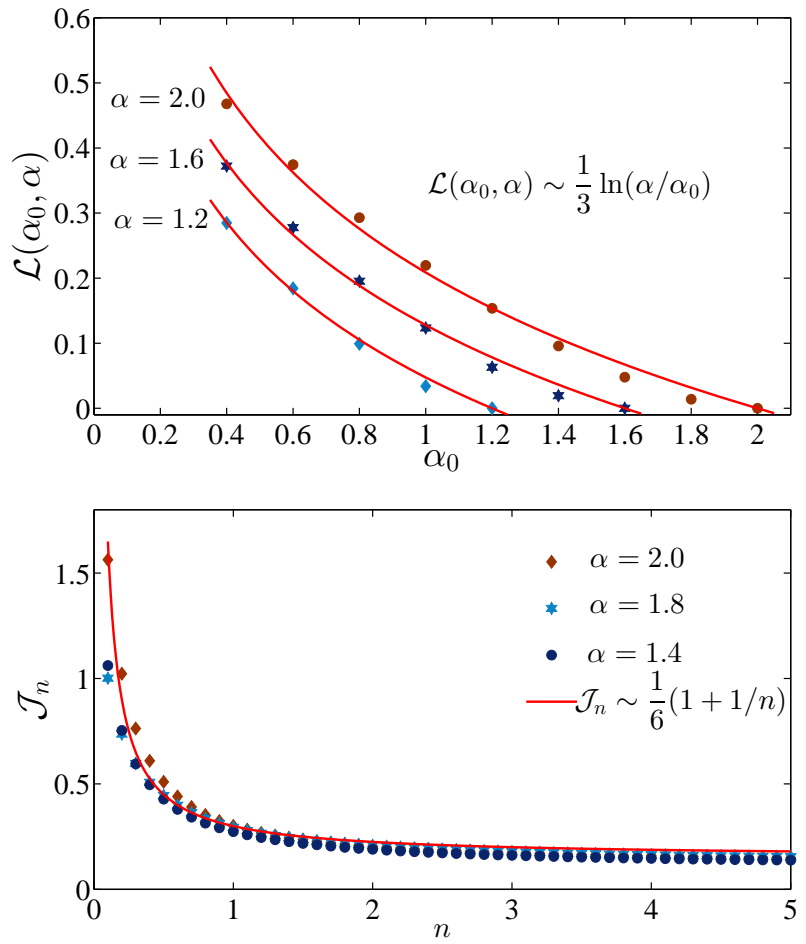

FIG. 15: (Color online) Top: The numerical estimates of the prefactor $\mathcal{L}\left(\alpha_{0}, \alpha\right)$ for different values $\alpha=2.0,1.6,1.2$ as a function of $\alpha_{0}$. The solid red lines correspond to $\mathcal{L}\left(\alpha_{0}, \alpha\right)=$ $\frac{1}{3} \ln \left(\alpha / \alpha_{0}\right)$. Bottom: The prefactor $\mathcal{J}_{n}$ for the dynamics of the Rényi entropy after quench from gapless power-law initial state (see Eq. (40) and (41)). The solid red line corresponds to $\frac{1}{6}(1+1 / n)$.

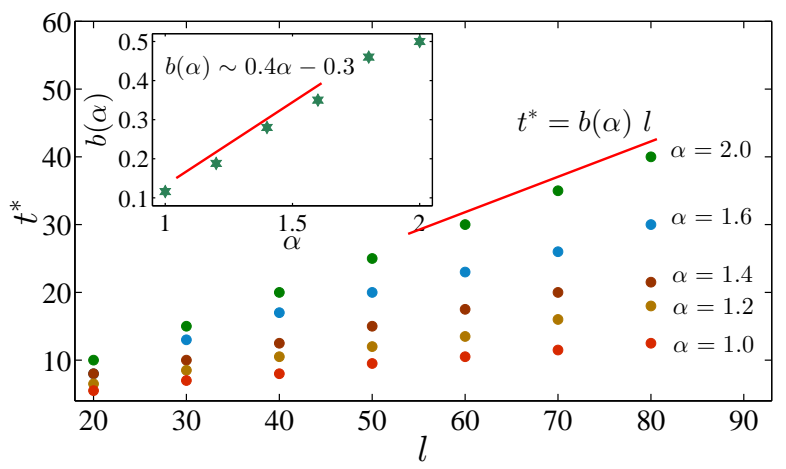

FIG. 16: (Color online) The relation between the saturation time $t^{*}$ and the subsystem size $l$ for different values $\alpha$. The solid red line represents $t^{*}=b(\alpha) l$. Inset: The prefactor $b(\alpha)$ as a function of $\alpha$ shows the linear behavior $b(\alpha) \simeq 0.4 \alpha-0.3$.

where $m$ is a positive real number. The interesting point about Eq. (42) is that one can easily find the correlation function $\left\langle\phi_{l} \phi_{l+n}\right\rangle \equiv K^{-1 / 2}(n)$ explicitly and the result is

$$
\begin{aligned}
K^{-1 / 2}(r) & =\int_{-\infty}^{\infty} e^{i q r}\left(|q|^{2}+m^{2}\right)^{-\alpha / 4} d q \\
= & \frac{\sqrt{\pi} 2^{(\alpha-6) / 4}}{\Gamma(\alpha / 4)}\left(\frac{r}{m}\right)^{(\alpha-2) / 4} \mathbf{K}[(\alpha-2) / 4, m r],
\end{aligned}
$$
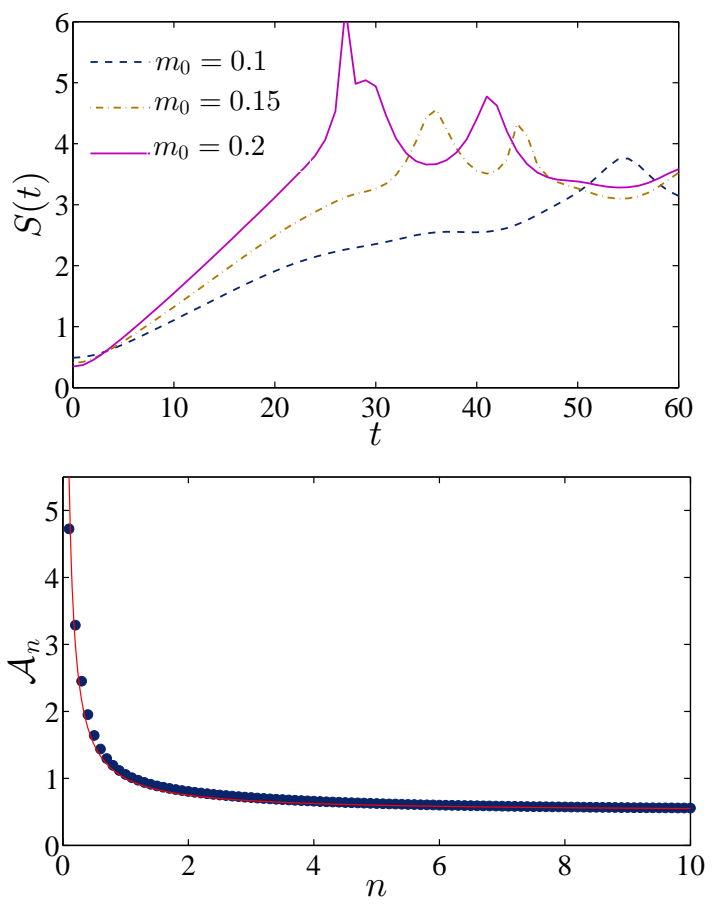

FIG. 17: (Color online) Top: Entanglement entropy dynamics $S_{A}(t)$ for long-range harmonic oscillator Eq. (42) with an exponential decaying correlation function. The total size of the system is $N=400$, the subsystem size $l=50$ and $\alpha=$ 1.5. Different lines corresponds to different values of the mass parameter $m_{0}$. Bottom: Prefactor $\mathcal{A}_{n}$ for dynamics of the Rényi entropy for the long-range harmonic oscillator system $(\alpha=1.5)$. The solid red line represents $\frac{1}{2}(1+1 / n)$.

where $\mathbf{K}$ is the modified Bessel function. The large distance asymptotic behavior of the correlation function is

$$
K^{-1 / 2}(r) \propto e^{-m r} / r^{(\alpha-4) / 4} .
$$

Above result leads to a finite spatial correlation length $\xi_{s}^{-1} \propto m$.

Using the technique of the previous sections, we provided the numerical test for the entanglement evolution $S_{A}(t)$ of the long-range harmonic oscillators $(\alpha \neq 2$ in Eq. (42)). In our study, initially $(t=0)$ the harmonic chain is massive $m_{0}>0$, and after the quench for $t>0$ the hamiltonian (42) is critical with $m=0$.

Our results for $\alpha>1$ clearly shows that the qualitative and quantitative behavior of $S_{A}(t)$ for $t<t^{*}$ are similar to those long-range systems with power-law correlation functions. For example we find numerically that the entanglement dynamics $S_{A}\left(t \ll t^{*}\right)$ in very short time have the same quadratic growth as the previous sections. A more careful analysis also shows that the $S_{A}(t)$ grows linearly in time until the saturation time $t^{*}$. Our numerical estimate of the linear coefficient $\mathcal{A} \sim \pi / 6$ was in agreement with the Eq. (34). In summary for the long-range harmonic oscillators with $\alpha>1$ we get the following re- 
sults:

$$
S_{A}(t) \equiv-\frac{c^{g}(\alpha)}{3} \log m_{0}+\left\{\begin{array}{ll}
\kappa_{2} t^{2} & t \ll 1 \\
\mathcal{A} m_{0}^{\alpha / 2} t & t<t^{*}
\end{array} .\right.
$$

In the above equation we checked that the prefactors $c^{g}(\alpha)$ and $\kappa_{2}$ are same as that in Eq. (34).

Now we can ask, what is the time-dependent behavior of Rényi entropy? We have seen that the results for $t<t^{*}$ is exactly the same as for the previous sections. As shown in Fig. (17) the agreement between the previous results given by Eq. (35) and Fig. (10) are fairly good.

Unfortunately, in the saturation regime it was difficult to study the scaling behavior of the $S_{A}(t \rightarrow \infty)$ and $S_{n}(t)$ with the mass parameter $m_{0}$ and the subsystem size $l$ because of the rapid oscillations in $S_{A}(t)$ and $S_{n}(t)$.

It is also straightforward to change the initial state for the harmonic system with strongly long-range couplings. In Fig .(18), we plot the time evolution of the entanglement entropy for the harmonic oscillators with very long-range interactions $(\alpha<1)$. Here we only find rapid oscillations around a constant value which depends to the subsystem size $l$ and mass parameter $m_{0}$. For short time as we expect, the $S_{A}(t)$ grows quadratically in time. The key observation here is that the initial state of the system completely changes the behavior of the entanglement dynamics for strongly coupled harmonic oscillators.

Finally let us now consider the maximum group velocity for the quasiparticles which produced after quantum quench from exponential decaying initial state to the critical point of the hamiltonian Eq. (42). When the system size $N$ is large enough, it is easy to verify that Eq. (42) leads to $v_{g}(k)=\frac{\alpha}{2}|k|\left[|k|^{2}+m^{2}\right]^{\alpha / 4-1}$ and one can obtain an exact result for the maximum group velocity $v_{g}^{\max } \propto m^{\alpha / 2-1}$. When the system is quenched to the critical point $(m=0), v_{g}^{\max }$ diverges and there is no maximum group velocity for the quasiparticles created during the quench. We emphasize that in the case where the long-range harmonic system is weakly coupled, there is no maximum group velocity but the $S_{A}(t)$ grows linearly with time. This means that in our long-range system, there is no direct signature of energy quasiparticles in the special behavior of the entanglement entropy growth.

\section{Unentangled initial state}

In this subsection we analyzed the case in which the harmonic oscillator system is initially (at $t=0$ ) prepared in a given unentangled state. Therefore, we begin with a chain in the absence of any couplings between two different sites of the chain and then quench the system to the critical point of the hamiltonian Eq. (28). In this case the initial entanglement entropy is zero $\left(S_{A}(t=0)=0\right)$.

We first consider the time evolution of the entanglement entropy of short-range harmonic oscillators with

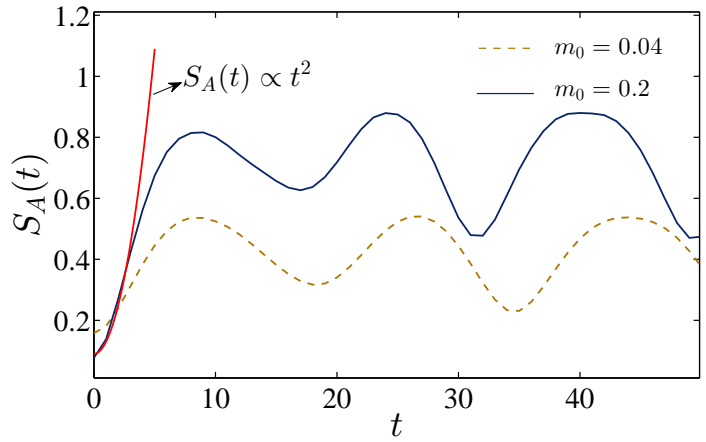

FIG. 18: (Color online) Entanglement entropy dynamics $S_{A}(t)$ for long-range harmonic oscillator Eq. (42) with strong coupling $(\alpha<1)$ which the initial state's correlation function decays exponentially. The total size of the system is $N=400$, the subsystem size $l=50$ and $\alpha=0.6$. Different lines corresponds to different values of the initial mass parameter $m_{0}$.

configuration $\mathfrak{g}_{1}$. We present results from numerical calculations of the von Neumann and Rényi entropies according to Eqs. (20) and (22), respectively. The evolution of von Neumann entropy $S_{A}(t)$ is shown in Fig. (19) which clearly showing that $S_{A}(t)$ exhibits linear behavior in time $t$ until it saturates at $t^{*}=l / 2$. However, some peaks appear in the linear regime for short periods of time which we believe that the peaks will disappear in $N \rightarrow \infty$ limit.

All the data of the time evolution of von Neumann and Rényi entropies are well fitted by

$$
S_{n}(t)=\left\{\begin{array}{cc}
\frac{\pi c_{n}}{6} t & t<l / 2 \\
\frac{\pi c_{n}^{\prime}}{12} l & t>l / 2
\end{array}\right.
$$

where in our numerical simulations, we found $c_{n}=\frac{1}{2}(1+$ $1 / n)$ and $c_{n}^{\prime}=\frac{1}{2}(1+1 / n)$. In Fig. (19), we show our numerical results for the prefactors $c_{n}$ and $c_{n}^{\prime}$.

We also studied time evolution of the Rényi entropy in the long-range harmonic oscillators with unentangled initial state. The resulting values of entanglement dynamics $S_{A}(t)$ for long-range harmonic chain with $\alpha=1.5$ is shown in Fig. (20). For weakly coupled long-range harmonic oscillators $1<\alpha<2$ we found that the best fit for the data is

$$
S_{n}(t)=\left\{\begin{array}{ll}
\mathcal{A}_{n} t & t<t^{*} \\
\mathcal{B}_{n} l & t>t^{*}
\end{array} .\right.
$$

Interestingly, we find that the best fit to $\mathcal{A}_{n}$ and $\mathcal{B}_{n}$ is $\frac{6 \mathcal{A}_{n}}{\pi}=\frac{12 \mathcal{B}_{n}}{\pi}=\frac{1}{2}(1+1 / n)$. The coefficients $\mathcal{A}_{n}$ and $\mathcal{B}_{n}^{\pi}$ as functions of $n$ are represented in Fig. (20). We then compute the saturation time $t^{*}$ which our numerical results clearly show that $t^{*}=\frac{4}{\alpha^{2}}(l / 2)^{\alpha / 2}$.

Finally, we should mention that the time evolution of the von Neumann entropy which the initial state is uncoupled and the final state is the critical state of the 

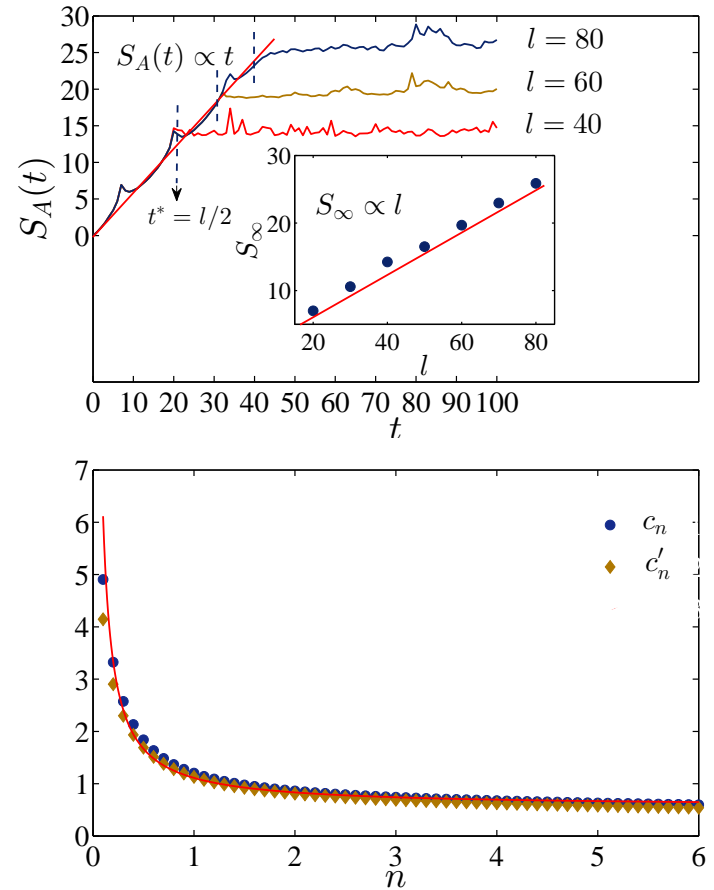

FIG. 19: (Color online) Top: The time evolution of the von Neumann entropy $S_{A}(t)$ in the short-range harmonic oscillators with the total size $N=500$ when the initial state is uncoupled. $S_{A}(t)$ starts from zero and grows linearly with time then saturates to the value $S_{\infty}$ which is a linear function of subsystem size $l$ (see inset). Bottom: The numerical estimates of the prefactors $c_{n}$ and $c_{n}^{\prime}$. The solid red line corresponds to $\frac{1}{2}(1+1 / n)$.

strongly long-range harmonic oscillator, follows a simple formula

$$
S_{A}(t)=\mathcal{P}(l, \alpha) \log t,
$$

where $\mathcal{P}(l, \alpha)=\mathcal{V}_{1}(\alpha)(l)^{\alpha / 2}+\mathcal{V}_{1}(\alpha)$ and the prefactors $\mathcal{V}_{1}$ and $\mathcal{V}_{2}$ are the same as those introduced in Sec. (V).

At this stage we should mention that our calculations show a universal behavior in the time evolution of the von Neumann and Rényi entropies for weakly coupled longrange harmonic oscillators $(1<\alpha<2)$. In other words initial state of the system does not change the behavior of the entanglement dynamics. It is worth mentioning that the prefactors of the linear parts of $S_{A}(t)$ and $S_{n}(t)$ in weak couplings regime $(1<\alpha<2)$ are not affected by the initial states of the system. Another interesting aspect to consider is that the entanglement dynamics for strongly coupled harmonic oscillators, identifying qualitatively different behavior as the initial state of the system varied.

\section{MUTUAL INFORMATION DYNAMICS}

In this section, we study the time evolution of the mutual information between two distant point $i$ and $j$ of the
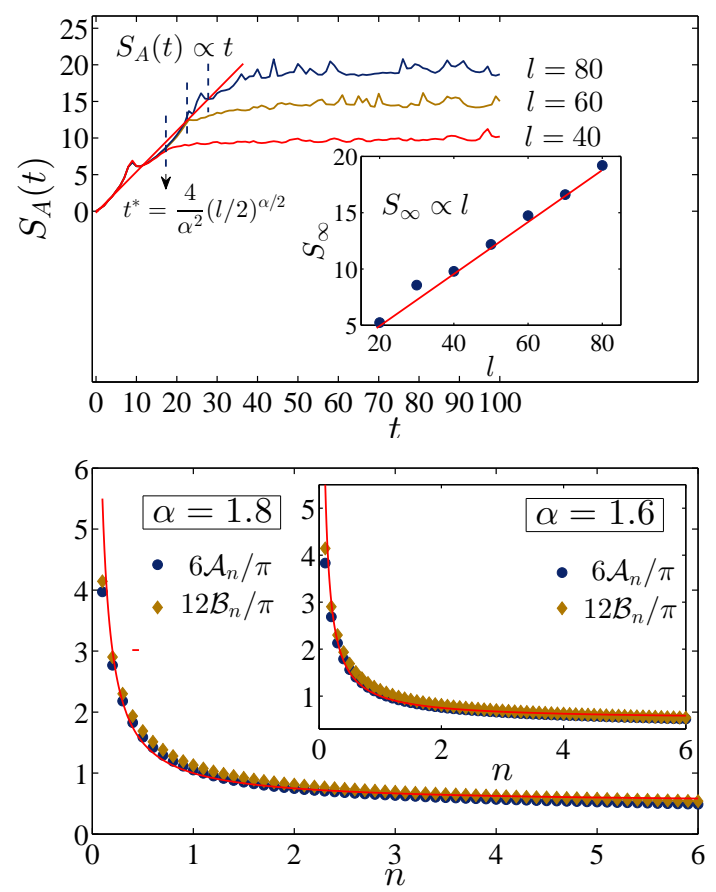

FIG. 20: (Color online) Top: The time evolution of the von Neumann entropy $S_{A}(t)$ in the long-range harmonic oscillators $(\alpha=1.5)$ with the total size $N=500$ when the initial state is uncoupled. $S_{A}(t)$ starts from zero and grows linearly with time then saturates to the values $S_{\infty}$ which is a linear function of subsystem size $l$ (see inset). Bottom: The numerical estimates of the prefactors $\mathcal{A}_{n}$ and $\mathcal{B}_{n}$ for different value $\alpha=1.6,1.8$. The solid red lines correspond to $\frac{1}{2}(1+1 / n)$.

harmonic chain with long-range couplings. The quantum mutual information is defined as follows

$$
I_{A_{1}, A_{2}}(t)=S_{A_{1}}(t)+S_{A_{2}}(t)-S_{A_{1} \cup A_{2}}(t),
$$

where $A_{1}$ and $A_{2}$ are the $i$-th and $j$-th points of the harmonic chain respectively. First, we consider the time evolution of the mutual information $I_{i, j}$ between two sites $i$ and $j$ of the chain of harmonic oscillators with short range interaction. In the Fig. (21) one can find the mutual information between two points of the chain while increasing the distance. Obviously one can find that the mutual information of two particular points with distance $|i-j|$, remains nearly zero for a period of time, until it suddenly changes to the non-zero value.

In the Fig. (21), we plot the time evolution of the mutual information between two points of the long-range harmonic oscillator with the separation distance $|i-j| \in$ $[10,60]$. It is interesting to note that the time evolution of the mutual information for this case is the same as the harmonic chain with short range interaction. This behavior is the key point to understand the linear growth of the entanglement entropy for the long-range harmonic systems with $1<\alpha \leq 2$.

In contrast with the previous cases, we observe that for $\alpha<1$ the qualitative behavior of the time evolution 

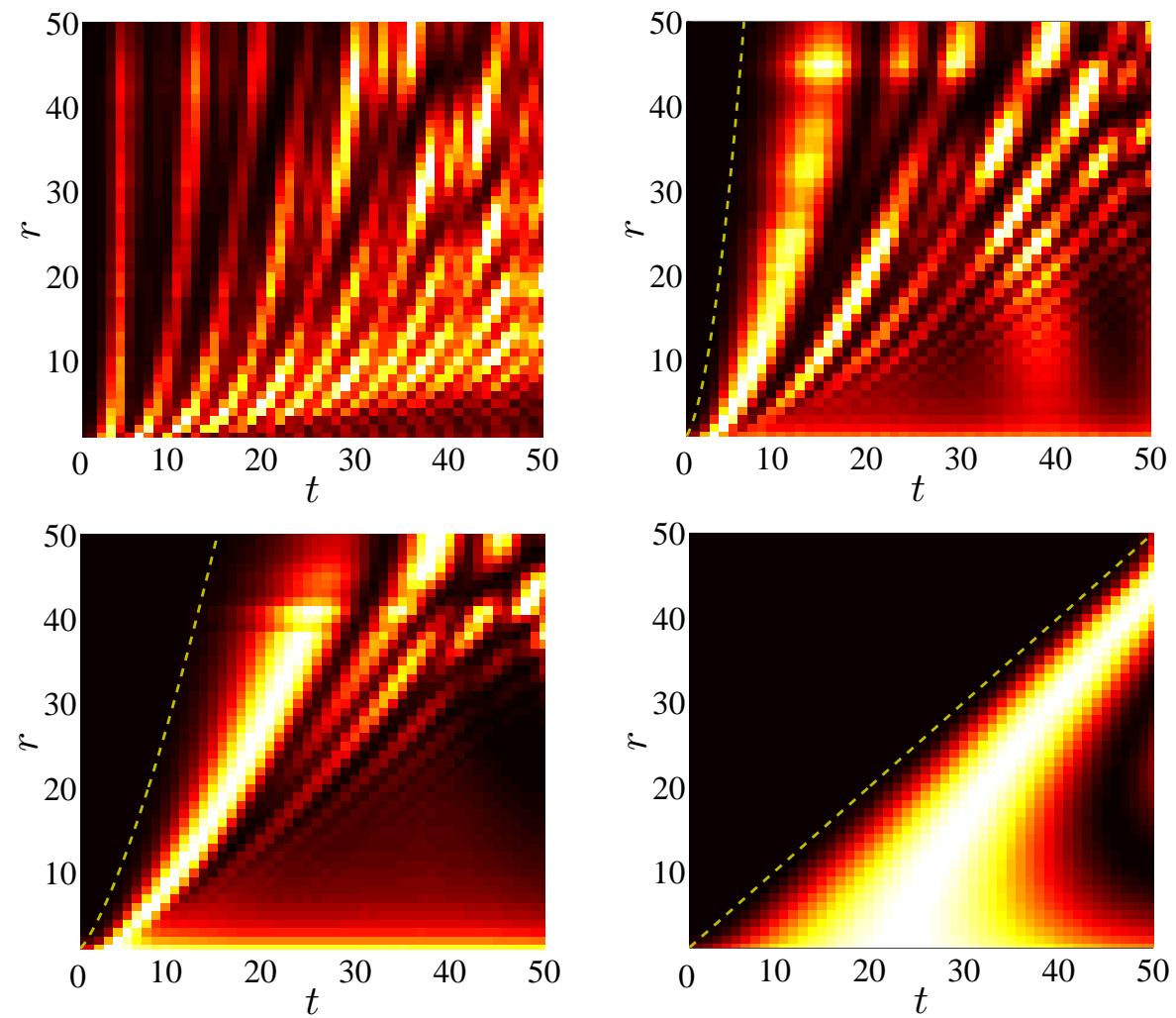

FIG. 21: (Color online) The density plot of mutual information $I_{i, j}$ between two sites with the separation distance $r=|i-j| / 2$ in short and long-range harmonic oscillators. The total size and initial mass parameter are equal to $N=200$ and $m_{0}=0.3$, respectively. Top left $(\alpha=0.4)$, right $(\alpha=1.0)$, and bottom left $(\alpha=1.4)$, right $(\alpha=2.0)$. Yellow (light Gray) regions correspond to larger value of $I_{i, j}$ and black regions correspond to smaller value of $I_{i, j}$. Dashed lines correspond to the $t_{I}^{*}=r^{\alpha / 2}$. At time $t_{I}^{*}$, the mutual information $I_{i, j}$ suddenly changes from zero to nonzero value. For $t<t_{I}^{*}$ the mutual information $I_{i, j}$ is zero and for $t>t_{I}^{*}$ it remains nonzero and oscillates due to finite-size effects.

of the mutual information between two distant points of the harmonic chain is completely different from the reported one for $\alpha>1$. The Fig. (21) shows a plot of the mutual information dynamics in harmonic chain with strong long-range couplings. It is clear that the mutual information grows rapidly from zero value at the beginning in which the behavior is independent of the distance separation of the two points.

In order to clarify the role of long-range couplings in the mutual information dynamics, we measure the time $t_{I}^{*}$ which before that time there is no mutual interaction between two points $i$ and $j$ of the harmonic chain. For later time $t>t_{I}^{*}$ the mutual information $I_{i, j}(t)$ changes to the non-zero value. In our simulation, as shown in Fig. (22) we observe

$$
t_{I}^{*}=r^{\alpha / 2},
$$

where $r=|i-j| / 2$ is the separation distance. It is worth mentioning that in Eq. (50) we find the same scaling behavior as Eq. (36).

It is worth mentioning that the similar regimes are reported in Ref. [44] for spin chains with variable range interaction. As it was previously mentioned, in Ref. [44]

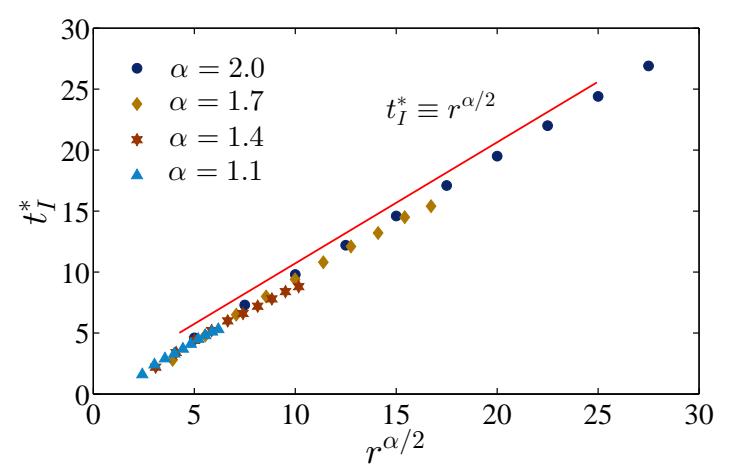

FIG. 22: (Color online) The numerical estimation for $t_{I}^{*}$ as a function of distance $r$. At time $t_{I}^{*}$, the mutual information $I_{i, j}$ of two particular points with distance $r=|i-j| / 2$ suddenly changes from zero to nonzero value.

they studied the time evolution of the mutual information between spins in the transverse field Ising model with long-range interactions $\left(J_{i, j} \propto|i-j|^{-\sigma}\right)$ which shows different regimes as a function of $\sigma$. It is interesting to note that they found that distant spins become entangled in- 
stantaneously for spin chains with strong couplings for $\sigma<1$. In contrast, we found that in our long-range harmonic chains, two different sites become entangled instantaneously when $\alpha<1(\sigma<2)$.

\section{CONCLUSION AND REMARKS}

We have investigated a detailed study of the time evolution of entanglement entropy and mutual information that results after a global quench in a chain of harmonic oscillators with short and long-range couplings. To do so first we have proposed an efficient method to numerically compute the time evolution of the von Neumann and Rényi entropies after a global quench in a general $d$ dimensional hamiltonian. For example we presented a detailed numerical evaluation for the entanglement dynamics in short-range harmonic oscillators. All our results are in good agreement with the theoretical predictions. Another interesting question to study is the time evolution of entanglement entropy in the harmonic chain with longrange couplings. We found that a regime of quadratic and linear entanglement entropy growth is present even for long-range coupled harmonic oscillators with $\alpha>1$. For the strongly coupled long-range systems with $\alpha<1$ we found a regime of logarithmic entanglement entropy growth. However, it is important to mention that it does not look like possible to explain this behavior with the energy quasiparticle picture of a global quench. Note that there is no maximum group velocity for those long-range systems that have been quenched to critical point of the system. We have also determined the time evolution of the entanglement entropy for different initial states. It is remarkable that the logarithmic growth of entanglement for strongly long-range coupled harmonic oscillators affected by the initial state. Focusing on the long-range harmonic oscillators we observed that the mutual information dynamics after the global quench exhibits numerous interesting dynamical behaviors. In contrast to the weakly coupled long-range harmonic oscillators, different sites become entangled instantaneously when the system is strongly coupled with $\alpha<1$.

For future studies, it could be of interest to study the time evolution of entanglement entropy and mutual information in harmonic oscillators with short and long-range couplings on higher dimensional lattices.

\section{Acknowledgments}

MGN kindly acknowledges numerous discussions with P. Calabrese, B. Doyon and M. van den Worm. MAR thanks FAPESP for financial support.

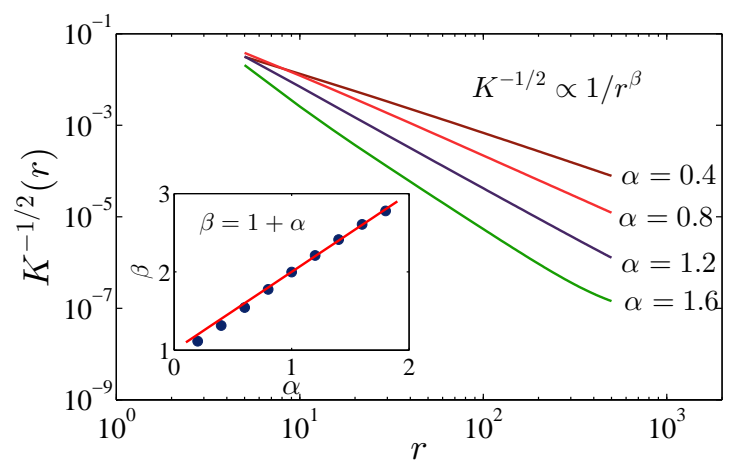

FIG. 23: (Color online) Numerical evaluation of $K^{-1 / 2}(r)$ vs $r$ for power-law initial state shows the scaling behavior $K^{-1 / 2}(r) \propto 1 / r^{\beta}$. Inset: Scaling exponent $\beta$ as a function of $\alpha$. The solid red line represents $\beta=1+\alpha$.

\section{Appendix A: Gapfull power-law initial state}

In this Appendix we discuss the numerical evaluation of the correlation length for the hamiltonian (28). We performed numerical integration Eq. (31) to find the correlation length. In the Fig. (23), $K^{-1 / 2}(r)$ is plotted vs $r$ using logarithmic scales. The figure shows that $K^{-1 / 2}(r)$ scales as $1 / r^{\beta}$. In the inset of Fig. (23) we depict the scaling parameter $\beta$ as a function of $\alpha$, which indicates $\beta=1+\alpha$. This power-law behavior specifies that the correlation length $\xi_{s} \equiv\left[\lim _{r \rightarrow \infty} \frac{\log (r)}{r}\right]^{-1}$ remains infinite even at $m \neq 0$.

\section{Appendix B: Details on numerical calculations}

The entanglement dynamics for coupled harmonic oscillators can be efficiently studied by the algorithm proposed in section III In this study a discrete scheme has been developed to simulate the entanglement dynamics of the short and long-range harmonic oscillator. We hereby, require a chain of $N$ harmonic oscillators described by Eq. (28). It was pointed out in the previous sections that to study the time evolution of the von Neumann and Rényi entropies, the system is prepared in the ground state of a massive hamiltonian $H\left(m_{0}\right)$ and at time $t=0$ the parameter $m_{0}$ is changed suddenly to a different value $m$. It is important to note that the Eqs. (25) and (26) have been proved for the system with size $N \rightarrow \infty$ and $m=0$. Here we will discuss the dependence of our results on finite $N$ and $m \neq 0$. In other words, it will be interesting to check whether and how the numerical results will be affected by the changes in chain size $N$ and mass parameter $m$.

Let us first consider the dispersion relation of the hamiltonian Eq. (28) as:

$$
\epsilon_{k}=\sqrt{[2-2 \cos (k a)]^{\alpha / 2}+m^{\alpha}},
$$

where $a$ is the lattice constant and $m$ is the mass parameter. In the previous sections the lattice constant had 

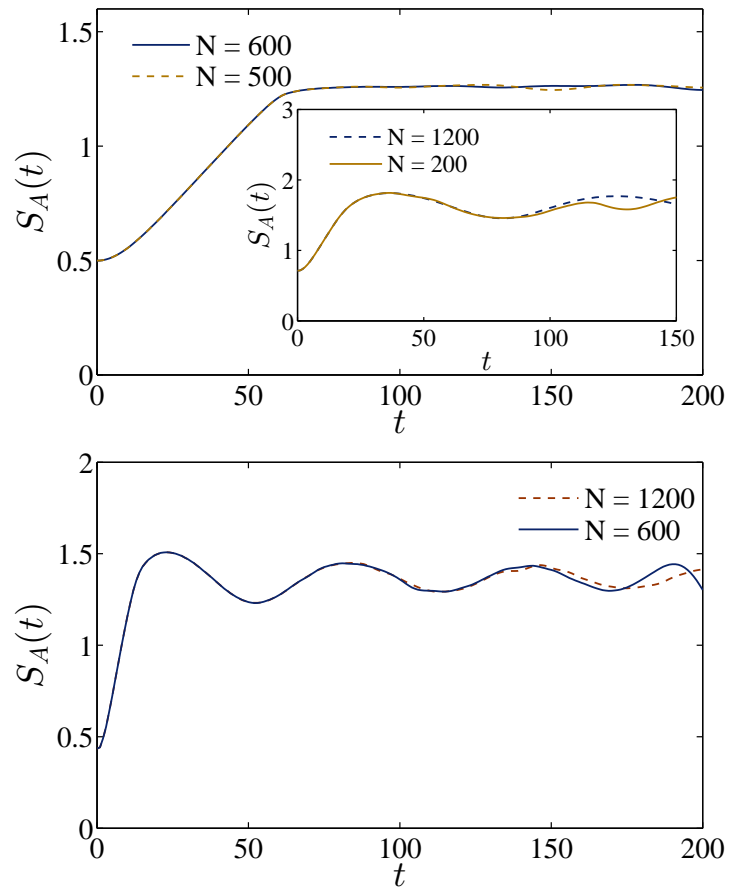

FIG. 24: (Color online) Top: Entanglement entropy dynamics $S_{A}(t)$ in short range harmonic oscillator with the configuration $\mathfrak{g}_{2}$ and different system size $N\left(l=30\right.$ and $\left.m_{0}=0.05\right)$. The same results for the configuration $\mathfrak{g}_{1}\left(l=30, m_{0}=0.12\right)$ shown in the inset. Bottom: $S_{A}(t)$ for long-range harmonic oscillator $(\alpha=1.5)$ with different system size $N$. Notice that the results do not depend on $N$.

been set equal to one.

Note that, because of the finiteness of the number of oscillators $N$, the energy spectrum is quantised, which the resulting quantization is $k=k_{n}=2 n \pi / N a$ and $n \in$ $[0, N-1]$.

It would also be instructive to extract the group velocity of the quasiparticle excitations which is defined as

$$
v_{g}\left(k_{n}\right)=d \epsilon_{k_{n}} / d k_{n}=\frac{\alpha\left|\sin \left(k_{n}\right)\right|^{\alpha-1}}{2 \sqrt{\left|\sin \left(k_{n}\right)\right|^{\alpha}+m^{\alpha}}} \cos \left(k_{n}\right)
$$

For the massless case $(m=0)$ the maximum group velocity can be found for $k \sim 0$. It is straightforward to calculate that $\lim _{k_{n} \rightarrow 0} v_{g}\left(k_{n}\right) \approx \frac{\alpha}{2}\left|k_{n}\right|^{\alpha / 2-1}$ which means that there is no maximum allowed velocity of the quasiparticles for $0<\alpha<2$. It is clear that for the short-range harmonic oscillators even for the system with finite size, the maximum group velocity of excitations is equal to unity $\left(\max \left[v_{g}(k)\right]=1\right)$.

If the system is gapped, based on the Eq. (B2) there is no maximum group velocity also for the harmonic chain with strong long-range couplings $(\alpha<1)$. We are mainly interested in the specific dependence of the maximum group velocity of excitations for those harmonic oscillators with $1 \leq \alpha \leq 2$, on the mass parameter $m$ and lattice constant $a$. The numerical results show that the maximum group velocity does not change with $N$ for large
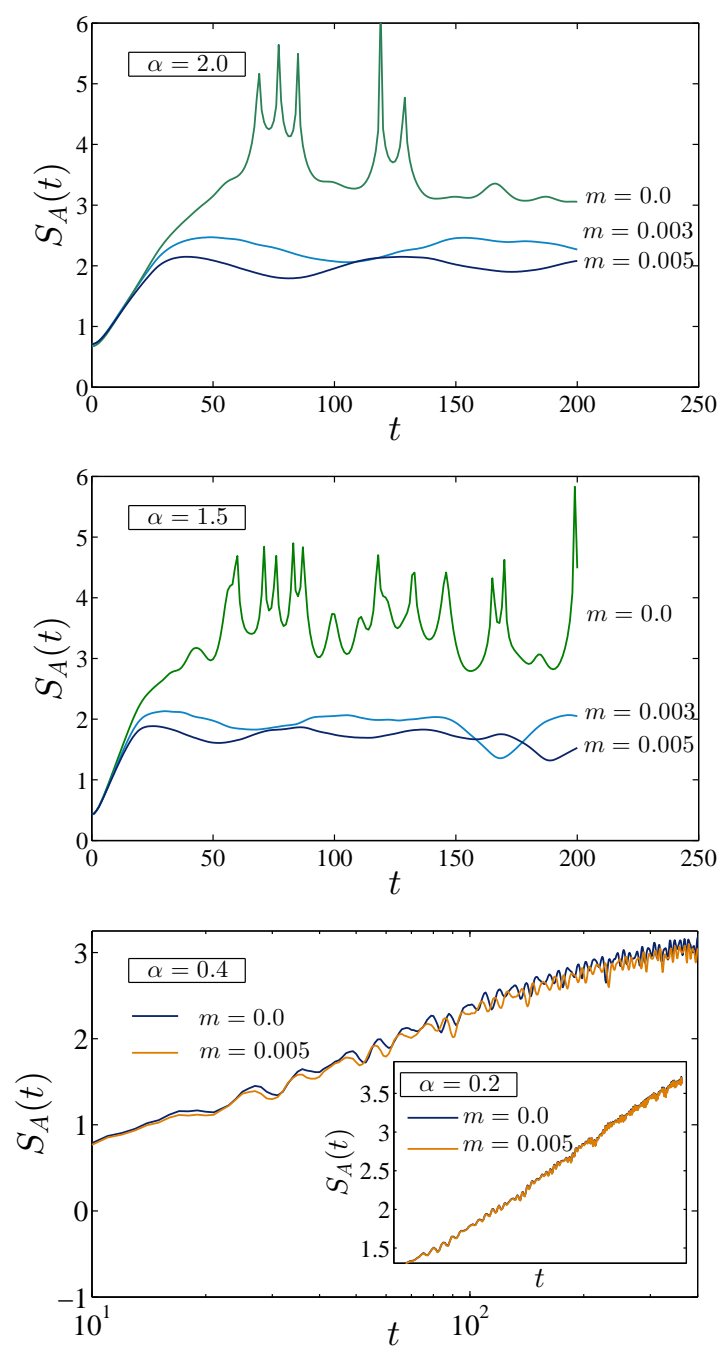

FIG. 25: (Color online) Top: Entanglement entropy dynamics $S_{A}(t)$ in short-range harmonic oscillator with the configuration $\mathfrak{g}_{1}$. The total size of the system is $N=500$, the mass parameter $m_{0}=0.12$ and the subsystem size $l=50$. Different lines corresponds to different values of the small mass parameter $m$. Middle: The same results for long-range harmonic oscillator with $\alpha=1.5$ with the configuration $\mathfrak{g}_{1}$, the system is $N=500$, the mass parameter $m_{0}=0.12$ and the subsystem size $l=50$. Bottom: Entanglement entropy dynamics $S_{A}(t)$ for long-range harmonic oscillators with strong coupling $\alpha=0.4$ and $\alpha=0.2$.

chain sizes. We checked numerically $N>500$ is large enough to find the $N$-independent value for the maximum group velocity. Then without changing the results one can safely analyses the maximum value of the Eq. (B2) in the limit $N \rightarrow \infty$. The Eq. (B2) becomes especially simple in the scaling limit:

$$
v_{g}=\frac{\alpha|k|^{\alpha-1}}{2 \sqrt{|k|^{\alpha}+m^{\alpha}}} .
$$

Then by simple algebra one can show that the maximum group velocity for the dispersion relation Eq. (B1) has 

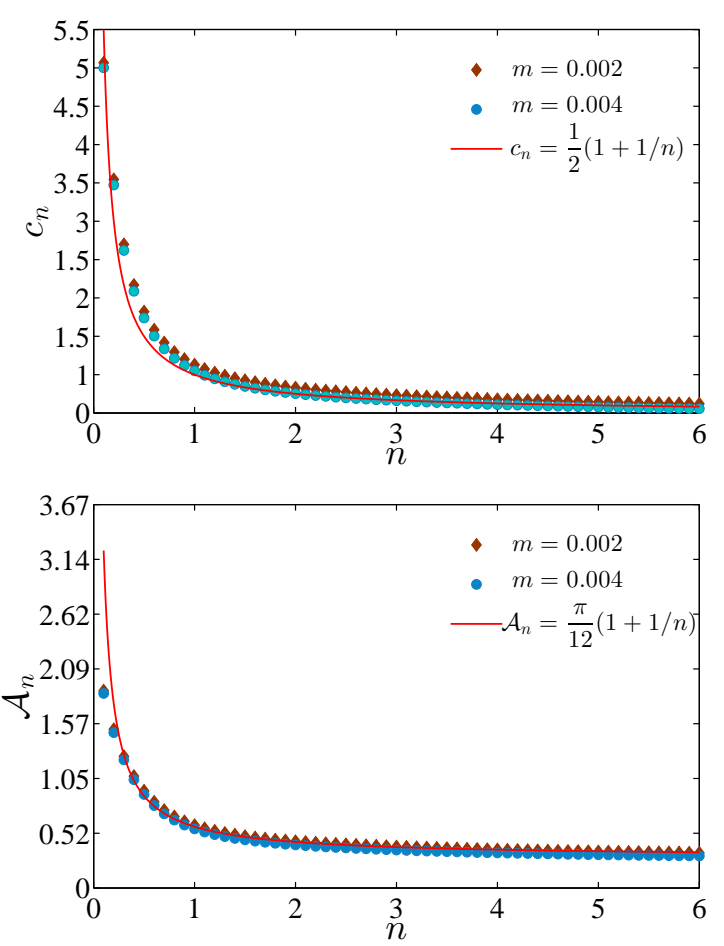

FIG. 26: (Color online) Top: The scaling prefactor $c_{n}$ for the entanglement dynamics in short-range harmonic oscillator before saturation regime (see Eq. (26)). Different symbols correspond to different values of the small mass parameter $m$. Bottom: The scaling prefactor $\mathcal{A}_{n}$ for the entanglement dynamics in long-range harmonic oscillator $(\alpha=1.5)$ before saturation regime (see Eq. (35)). Different symbols correspond to different values of the small mass parameter $m$.

the following form:

$$
v_{g}^{\max }=\frac{\alpha}{2}(2 / \alpha-1)^{1 / 2}\left(\frac{\alpha-1}{1-\alpha / 2}\right)^{\frac{\alpha-1}{\alpha}} m^{\alpha / 2-1} .
$$

Let us now consider the $m$ and $N$ dependence in time evolution of the entanglement entropy. In Fig. (24) we provide an examples of typical behavior of entanglement dynamics $S_{A}(t)$ for short range harmonic oscillator with configurations $\mathfrak{g}_{1}$ and $\mathfrak{g}_{2}$ and different values of the system size $N$. It is not surprising that our results are independent of $N$. As shown in the same figure the entanglement dynamic for harmonic oscillators with long-range couplings is $N$ independent.

It is important to note that when the the system is very large and the sub system is a small portion with length $l\left(\mathfrak{g}_{1}\right)$ our numerical results for $m=0$ show many oscillations in the saturation regime (see Fig. (25)). Therefore it is very hard to analysis the entanglement dynamics after saturation regime. The parameter $m \ll m_{0}$ has been chosen to decrease these oscillations. Figure (25) shows the entanglement dynamics $S_{A}(t)$ in the case which $m_{0}=0.12$ and $m \neq 0$. Let us now discuss the role of non-zero $m$ on the final results. It is important to note that we are not allowed to choose arbitrary value
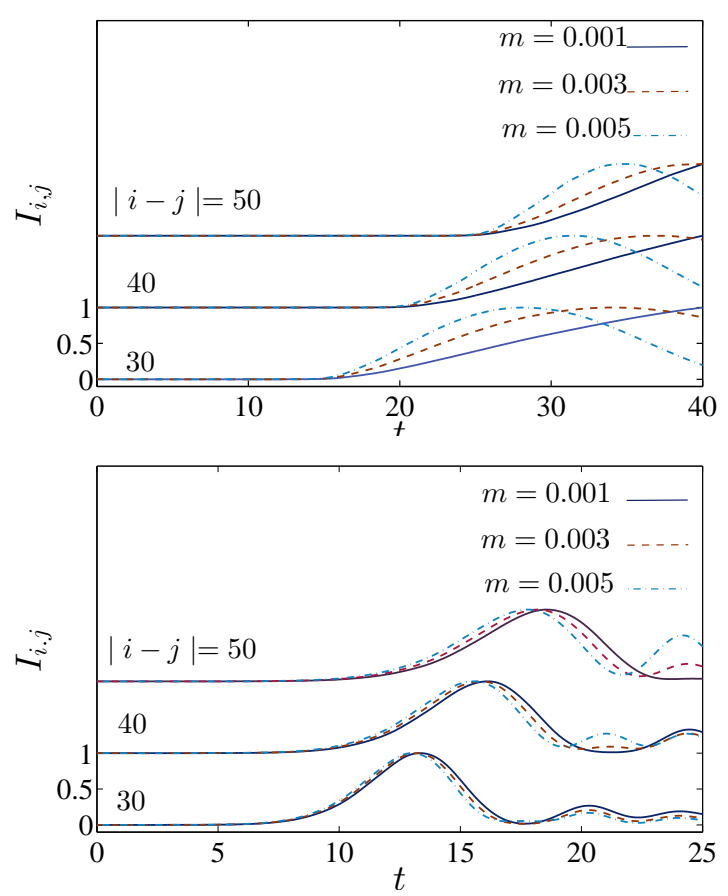

FIG. 27: (Color online) Top: The time evolution of the mutual information $I_{i, j}$ between two sites $i$ and $j$ in short range harmonic oscillators with the total size $N=200$, mass parameter $m_{0}=0.3$ and different values of the small parameter $m$ and distance $|i-j|$. Bottom: The same results for long-range harmonic oscillator $(\alpha=1.5)$.

for $m$. First, it should be smaller than the initial mass parameter $m_{0}$. Secondly one can choose $m \sim \mathcal{O}(1 / N)$ which $N$ is the system size. It is because of the finitesize effect in the the correlation length $\xi$. However, the correlation length is expected to diverge at critical point $(m=0)$, for a finite system it is comparable to the system size $\xi \propto m^{-1} \sim \mathcal{O}(N)$. We would also like to point out that all our results are consistent with this picture as long as $m \ll m_{0}$ and $m \sim 1 / N$. It is worth mentioning that the results were affected significantly by enough amount of mass term $(m \gg 1 / N)$. This means that the entanglement dynamics is independent of the maximum group velocity Eq. (B⿺辶) for $m \gg 1 / N$. It is worth mentioning that the same results observed for the harmonic chain with long-range couplings as well as short-range harmonic oscillators. In Fig. (26) we compare the numerical results of the prefactors $c_{n}$ and $\mathcal{A}_{n}$ (see Eq. (26) and (35) respectively) for different values of small mass parameter $m$ after quench. It is clear that the numerical results are $m$-independent.

Finally we would like to note that we did the same calculations for the mutual information dynamics $I_{i, j}(t)$ between two point $i$ and $j$ of the lattice sites. Note that for different values of the chain length $N$ we got the previous results, as it should and the results were independent from the system size $N$. In order to show the validity and reliability of our results, in Fig. (27) we report the 
time dependent mutual information results for the short and long-range harmonic oscillator with fixed values of $m_{0}=0.3$ and different values of the the parameter $m$. It is evident that the starting point which the mutual information changes from zero to non-zero value, is independent from $m$. We again found the same result $t_{I}^{*}$ for the different values of the small parameter $m$ which is clearly obeys the prediction $t_{I}^{*}=r^{\alpha / 2}$ where $r=|i-j| / 2$.
[1] C. Holzhey, F. Larsen and F. Wilczek, Nucl Phys. B 424, 443 (1994)

[2] G. Vidal, J. I. Latorre, E. Rico, and A. Kitaev, Phys. Rev. Lett. 90, 227902 (2003)

[3] V. Vedral, Rev. Mod. Phys. 74.1, 197 (2002)

[4] P. Calabrese and J. Cardy, J. Stat. Mech. P06002 (2004)

[5] L. Amico, R. Fazio, A. Osterloh, and V. Vedral, Rev. Mod. Phys. 80, 517 (2008)

[6] P. Calabrese and J. Cardy, Journal of Physics A: Mathematical and Theoretical 42(50), 504005 (2009)

[7] J. Eisert, M. Cramer and M. B. Plenio, Rev. Mod. Phys. 82, 277 (2010)

[8] K. Mallayya, R. Tibrewala, S. Shankaranarayanan and T. Padmanabhan, Phys. Rev. D 90, 044058 (2014)

[9] S. Ghosh and S. Shankaranarayanan, Phys. Rev. D 86, 125011 (2012)

[10] M. Srednicki, Phys. Rev. Lett 71666 (1993)

[11] Samuel L. Braunstein, Saurya Das and S. Shankaranarayanan, JHEP 1307 (2013) 130

[12] H. Casini and M. Huerta, J.Phys.A 42:504007 (2009)

[13] T. Nishioka, S. Ryu and T. Takayanagi, Journal of Physics A: Mathematical and Theoretical, 42 (50), 504008 (2009)

[14] J. I. Latorre, R. Orús, E. Rico and J. Vidal, Phys. Rev. A, 71 (2005) 064101; T. Barthel, S. Dusuel and J. Vidal, Phys. Rev. Lett., 97 (2006) 220402; S. Dusuel and J. Vidal, Phys. Rev. B, 71 (2005) 224420; M. Filippone, S. Dusuel and J. Vidal, Phys. Rev. A 83, 022327 (2011)

[15] R. Orús, S. Dusuel and J. Vidal, Phys. Rev. Lett. 101 (2008)

[16] W. Dür, L. Hartmann, M. Hein, M. Lewenstein and H. J. Briegel, Phys. Rev. Lett. 94, 097203 (2005).

[17] T. Koffel, M. Lewenstein and L. Tagliacozzo, Phys. Rev. Lett. 109, 267203 (2012)

[18] J. Eisert and T. J. Osborne, Phys. Rev. Lett. 97, 150404 (2006)

[19] M. A. Rajabpour and S. Sotiriadis, arXiv:1409.6558 (2014)

[20] A. Dutta and J. K. Bhattacharjee, Phys. Rev. B 64, 184106 (2001)

[21] T. Blanchard, M. Picco, M. A. Rajabpour, EPL 101, 56003 (2013)

[22] M. G. Nezhadhaghighi and M. A. Rajabpour, EPL 100, 60011 (2012)

[23] M. G. Nezhadhaghighi and M. A. Rajabpour, Phys. Rev. B 88, 045426 (2013)

[24] L. Bombelli, R. K. Koul, J. Lee, and R. D. Sorkin, Phys. Rev. D 34, 373 (1986)

[25] I. Peschel, J. Phys. A: Math. Gen. 36, L205 (2003)

[26] N. Shiba and T. Takayanagi, JHEP 1402, 033 (2014)

[27] D. W. Pang, Phys. Rev. D 89, 126005 (2014)

[28] S. Hofferberth, I. Lesanovsky, B. Fischer, T. Schumm, and J. Schmiedmayer, Nature, 449(7160), 324-327 (2007); S. Trotzky, Y. A. Chen, A. Flesch, I. P. McCulloch, U. Schollwöck, J. Eisert, and I. Bloch, Nature Physics, 8(4),
325-330 (2012); I. Bloch, J. Dalibard, and W. Zwerger, Rev. Mod. Phys, 80(3), 885 (2008); A. Polkovnikov, K. Sengupta, A. Silva, and M. Vengalattore, Rev. Mod. Phys., 83(3), 863 (2011).

[29] P. Calabrese and J. Cardy, J. Stat. Mech.: Theor. and Exp. P04010 (2005)

[30] J. Abajo-Arrastia, J. Aparicio and E. Lopez, JHEP 1011, 149 (2010)

[31] H. Liu and S. J. Suh, Phys. Rev. Lett. 112, 011601 (2014)

[32] M.B. Plenio, J. Hartley and J. Eisert, New J. Phys. 6, 36 (2004)

[33] A. Perales and M. B. Plenio, J. Opt. B: Quantum Semiclass. Opt. 7, S601-S609 (2005)

[34] F. Ghahari, V. Karimipour and R. Shahrokhshahi, Physics Letters A 363.4 (2007)

[35] H. Kim and D. A. Huse, Phys. rev. lett. 111, 127205 (2013)

[36] R. G. Unanyan and M. Fleischhauer, Phys. Rev. A 90, 062330 (2014).

[37] G. De Chiara, S. Montangero, P. Calabrese and R. Fazio, J. Stat. Mech. 0603, P001 (2006).

[38] V. Alba and F. Heidrich-Meisner, arXiv:1402.2299 (2014)

[39] E. Canovi, E. Ercolessi, P. Naldesi, L. Taddia and D. Vodola, Phys. Rev. B, 89(10), 104303. (2014)

[40] J. Eisert and T. J. Osborne, Phys. Rev. Lett. 97, 150404 (2006)

[41] E. H. Lieb and D. W. Robinson, Communications in Mathematical Physics 28, 251257 (1972)

[42] M. Cramer, A. Serafini, and J. Eisert, Quantum information and many body quantum systems, 5173, CRM Series, Ed. Norm., Pisa, (2008)

[43] S. Bravyi, M. B. Hastings, and F. Verstraete, Phys. Rev. Lett. 97, 050401 (2006)

[44] J. Schachenmayer, B. P. Lanyon, C. F. Roos, and A. J. Daley, Phys. Rev. X 3, 031015 (2013)

[45] P. Richerme, Z. X. Gong, A. Lee, C. Senko, J. Smith, M. Foss-Feig, S. Michalakis, A. V. Gorshkov, C. Monroe, Nature 511, 198201 (2014)

[46] P. Jurcevic, B. P. Lanyon, P. Hauke, C. Hempel, P. Zoller, R. Blatt, and C. F. Roos, Nature 511, 202205 (2014)

[47] B. Nachtergaele, Y. Ogata, and R. Sims, J. Stat. Phys. 124, 1 (2006)

[48] M. B. Hastings and T. Koma, Commun. Math. Phys. 265, 781 (2006)

[49] P. Hauke and L. Tagliacozzo, Phys. Rev. Lett. 111, 207202 (2013)

[50] J. Eisert, M. van den Worm, S. R. Manmana, and M. Kastner, Phys. Rev. Lett. 111, 260401 (2013)

[51] Z. X. Gong, M. Foss-Feig, S. Michalakis and A. V. Gorshkov, Phys. Rev. Lett. 113, 030602 (2014)

[52] D. Métivier, R. Bachelard and M. Kastner, Phys. Rev. Lett. 112, 210601 (2014)

[53] C. Callan, and F. Wilczek, Phys. Lett. B 333, 55 (1994). 